\title{
$46310=$ 6360
}

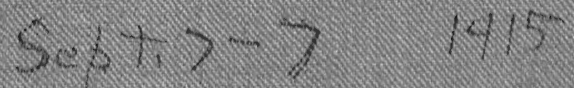




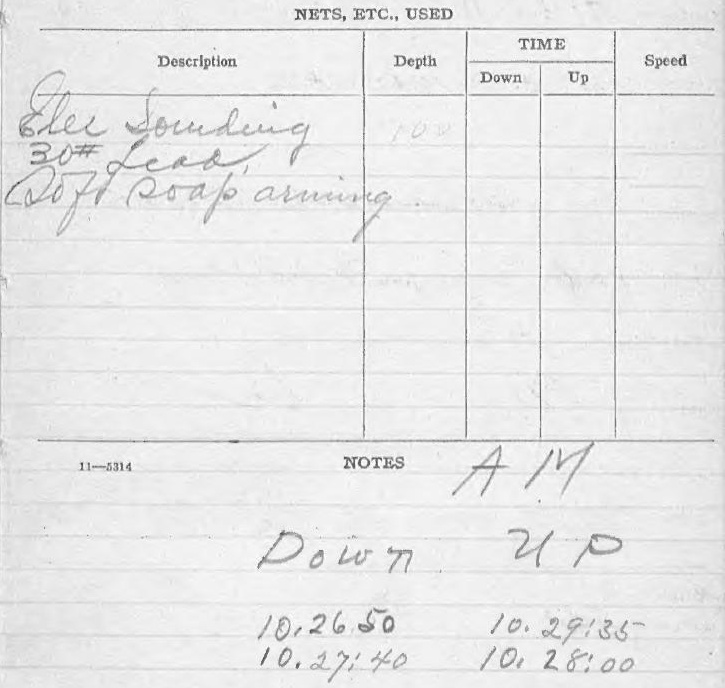

Chast 6100 
NETS, ETC., USED

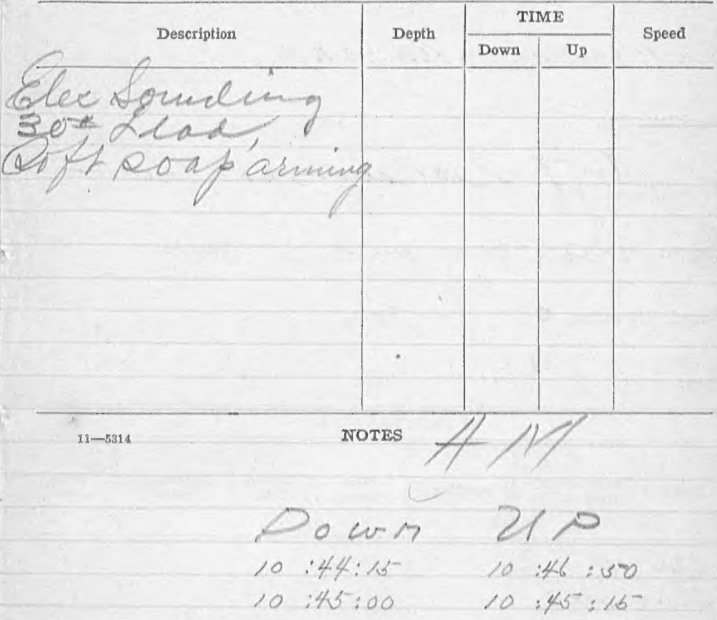

Prition: Till amosk Anad dy hit hears N64\%" (ture) diataut 26.6 mile Chart 6100 
NETS, ETC., USED

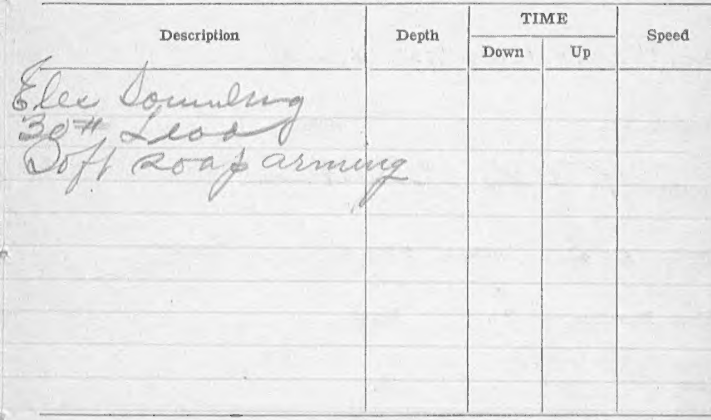

$11-5314$ NOTES
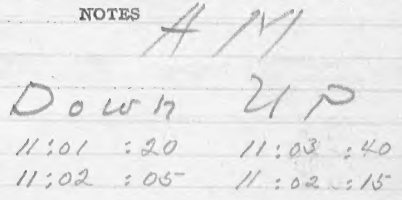

Proitiou! Tillamoost Head 2igut hears $N 63^{\prime} / 2^{\circ} \mathrm{E}$ (ture) distant 25.8 miles chart 6100 
NETS, ETC., USED

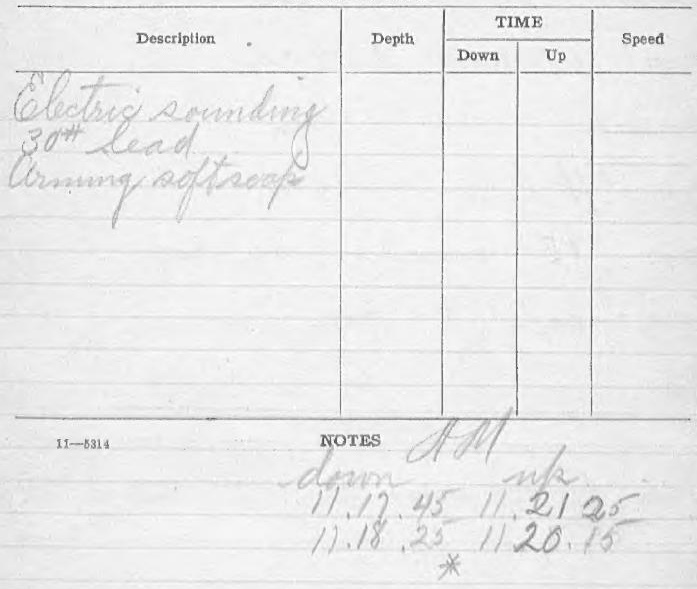

Position:Tillamosed Nead Light hears $N 6212^{\circ} E$ distant 25:3 suiles chart 6/00 
NETS, ETC., USED

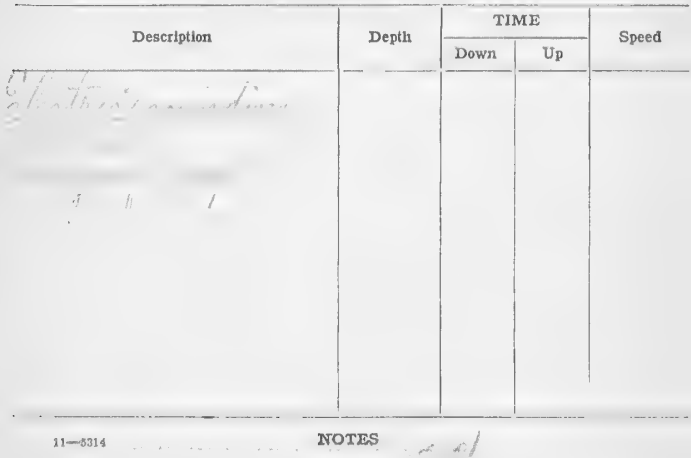

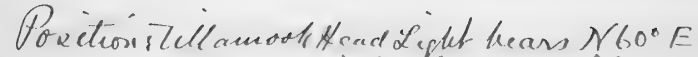
(true) distant 23,8 mules chart 6100 
Hour: $\{2,0 ; 3\}$.

Position: Lat.

Long.

\title{
Locality:
}

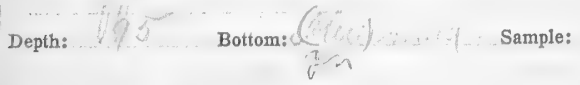

\begin{abstract}
Wind: Direction, $\ldots$ Force, ........
\end{abstract}

Sea: ....... Sky:

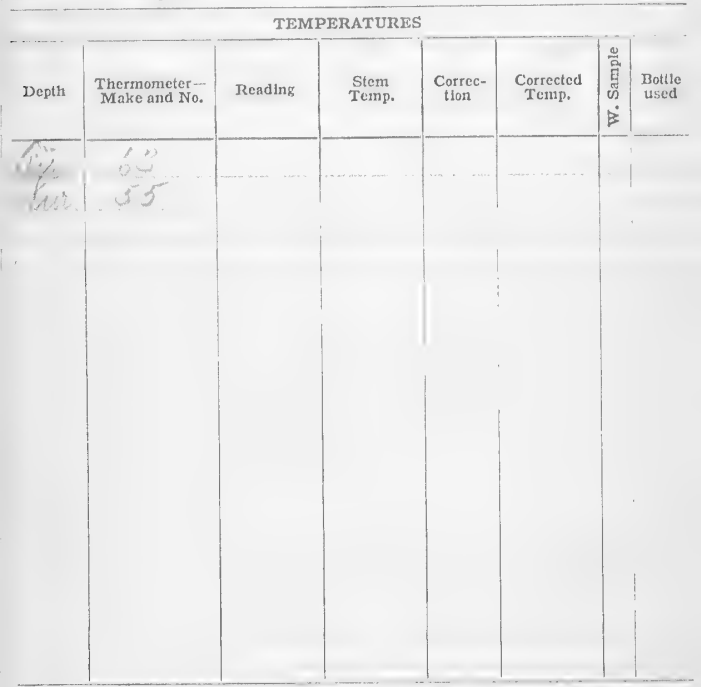

CURRENTS

\begin{tabular}{l|l|l|l|l|l|}
\hline Depth & Tide & Time down & Revolutions & Speed & Direction \\
\hline & & & & \\
& & & &
\end{tabular}




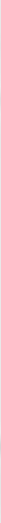

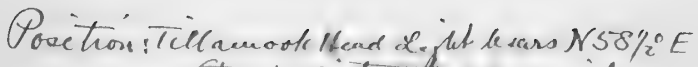
Chart (ture) diat and 25.1 muilechast 6100 
Station No. $463 / 7$ Date: 619

Hour: $12: 42: 10$ P.M. to $12: 44: 15=$ P. M.

Position: Lat.

Long.

Iocality:

Depth: gg ....... Bottom: gen $21, \ldots$ Sample:

Wind: Direction,

Sea: $\ldots+\ldots l / \ldots+\ldots \ldots+$ Sky: $=$

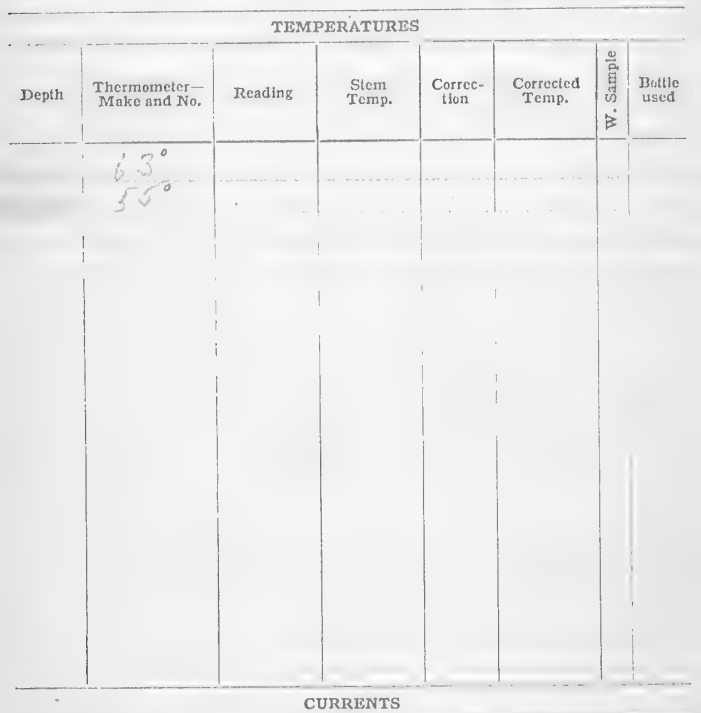

\begin{tabular}{l|l|l|l|l|l|l}
\hline Depth & Thour & Tide & Time down & Revolutions & Speed & Direction \\
\hline & & & & & \\
\hline & & & & & \\
& & & & & \\
\hline & & & & & \\
\hline
\end{tabular}


Station No.t $63 / 8$ Date: $+2 / 1915$ Hour: 12:6:3:50 P. M. to $: 0.0: 00$ P.M.

Position: Lat.

Long.

Locality: $\left(f_{i}\right), C, \cdots, e^{4}$

Depth: $10 \%$ feth Bottom: tim gig.

Sample:

Wind: Direction, $J$.
Sea:
Sky: fo

TEMPERATURES

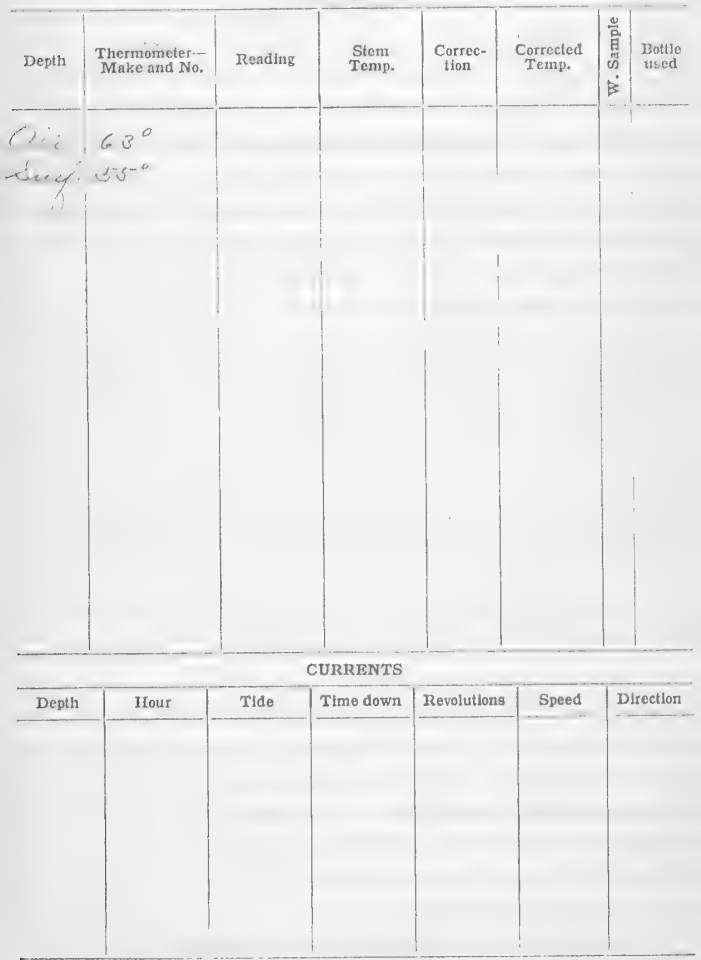

Color of water:

Transparency:

REMARKS:

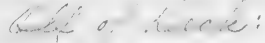

11-5i14 
Station No. H $68 / 9 \quad$ Date: $\quad 2 \ldots 1915$

Hour: $/: 13$ is $=P . M$. to $\quad 1: 16: 00 P . M$.

Position: Lat.

Long.

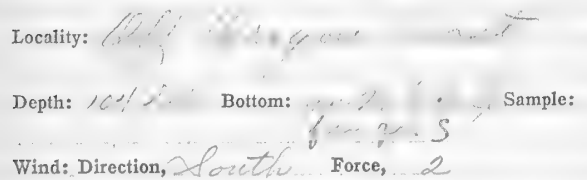

Sea: . Q

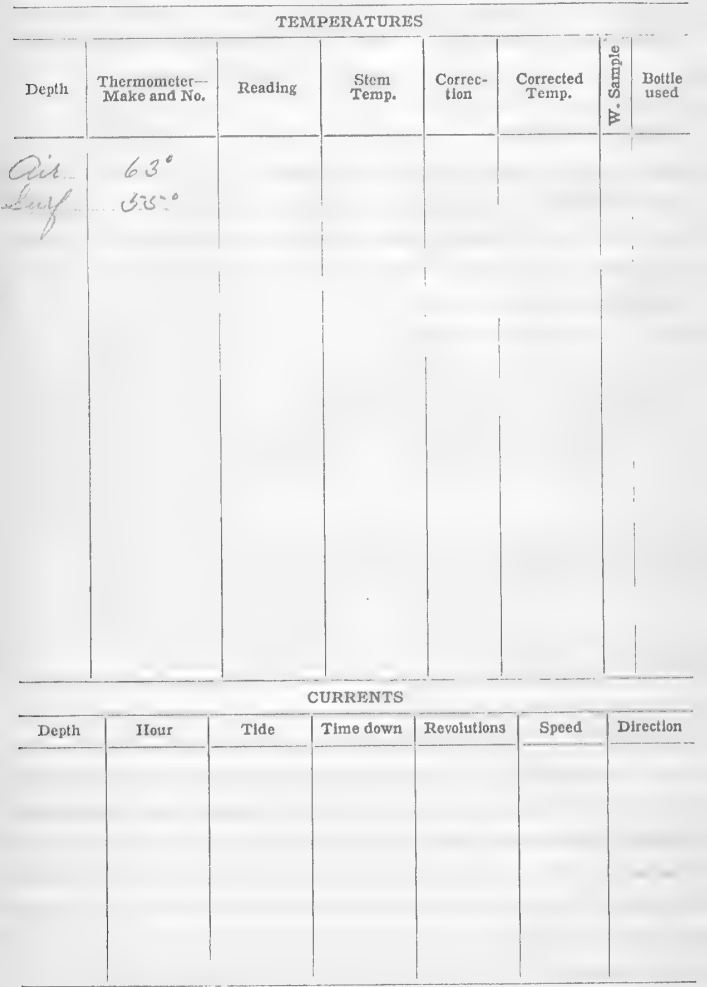

Color of water: inc

Transparency:

REMARKS:

$11-5314$ 
NETS, ETC., USED

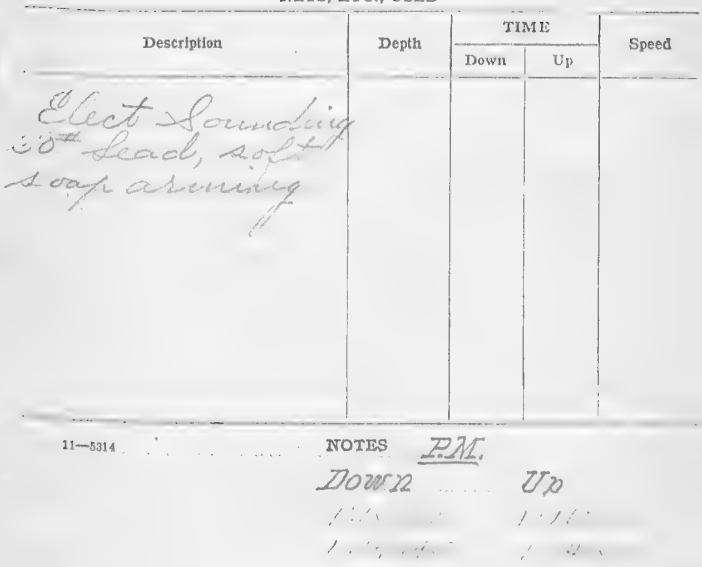

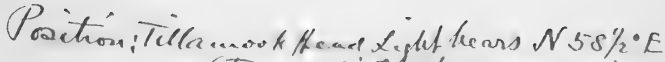
(Tue) risfdet 26.2 mide. Cllast 6100 
Hour: $1: 29: 40$ P.M. to $1: 31: 3=0 . M$.

\section{Position: Lat.}

Long.

\section{Locality:}

Depth: $101, ;$, Bottom:

Wind: Direction,

Sea:

Sea: 72

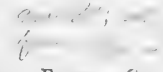

Force, -

\section{Sample:}

Sky:

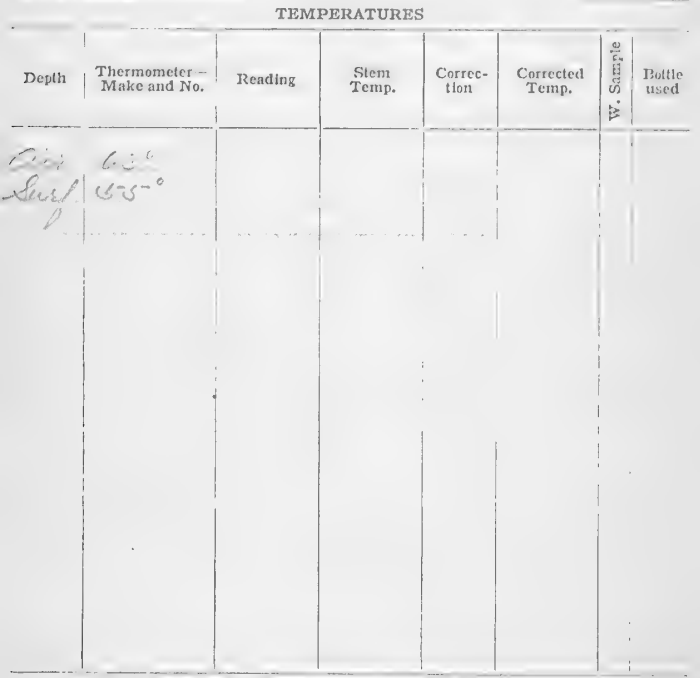

\section{CURRENTS}

\begin{tabular}{l|l|l|l|l|l|l}
\hline Depth & Hour & Tide & Time down & Revolutions & Speed & Direction \\
\hline & & & & & \\
& & & & & & \\
& & & & & & \\
\hline
\end{tabular}

\section{REMARIS:}


Station No. $/ 3$ \& 1

Date:

Hour: 1 , $4: 30$ P.M. to $1: 48: 45$ D. M.

Position: Lat.

Locality:

Depth: /N, Bottom:
Long.

Sample:

Wind: Direction, if oec z Force, 3

Sea: CLC, Slry: - Cre

TEMPERATURIS

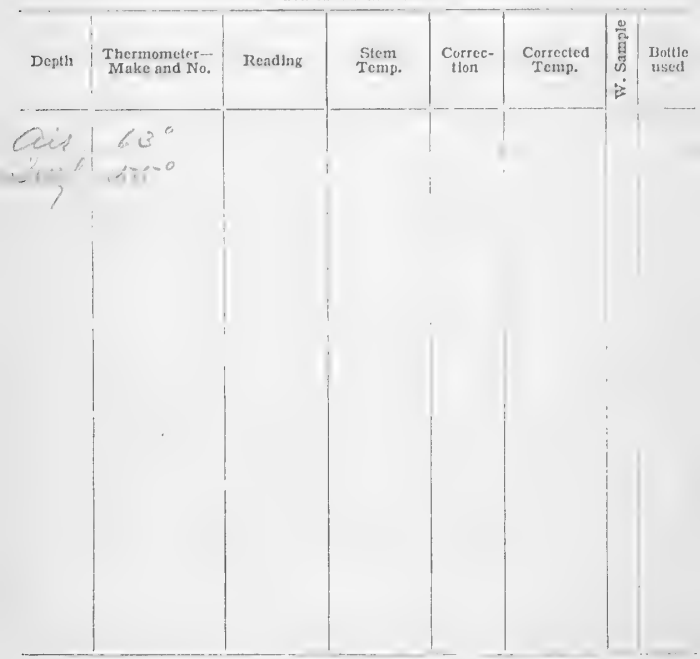

CURRENTS

\begin{tabular}{l|l|l|l|l|l|l}
\hline Depth & Hour & Tide & Time down & Revolutions & Speed & Direction \\
\hline & & & & & \\
\hline & & & & & \\
& & & & & & \\
& & & & & & \\
\hline
\end{tabular}

Color of water:

Transparency:

REMARKS:

$11-8314$ 
NETS, ETC., USED

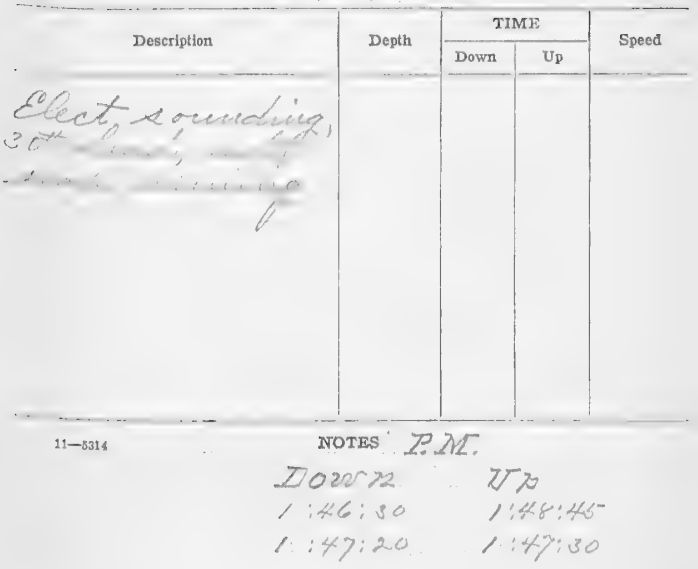

Prvition: Tillanoot Nend dight hears $N 5 g^{\circ} \mathrm{E}$ Chast-6,100 


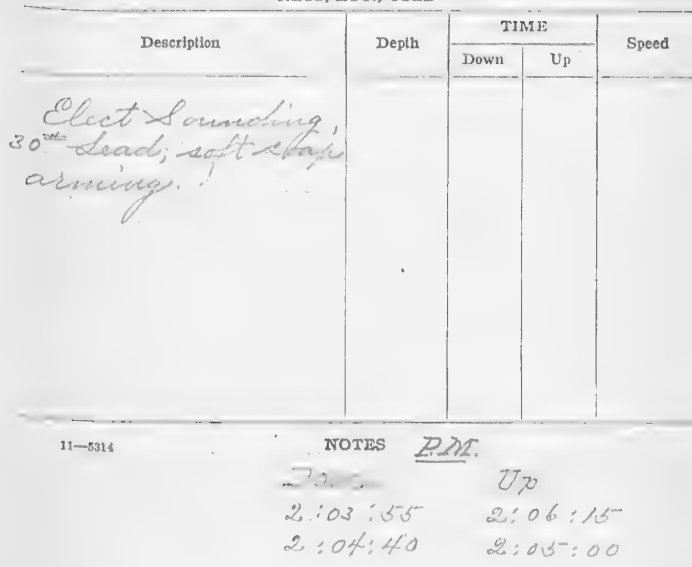

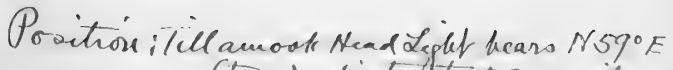
(tuc) dielciely 27,5 m.ine chant 6100 
Hour: $2: 21 ; 10$ P.M. to $2: 20 ; 0 ;$ P.M.

Position: Lat.

Long.

\section{Locality:}

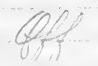

Depth:

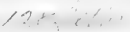

Bottom:

Wind: Direction,
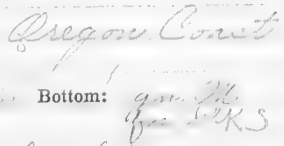

Force, 3

Sky: acper

Sample:
Sea:

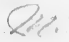

TEMPERATURES

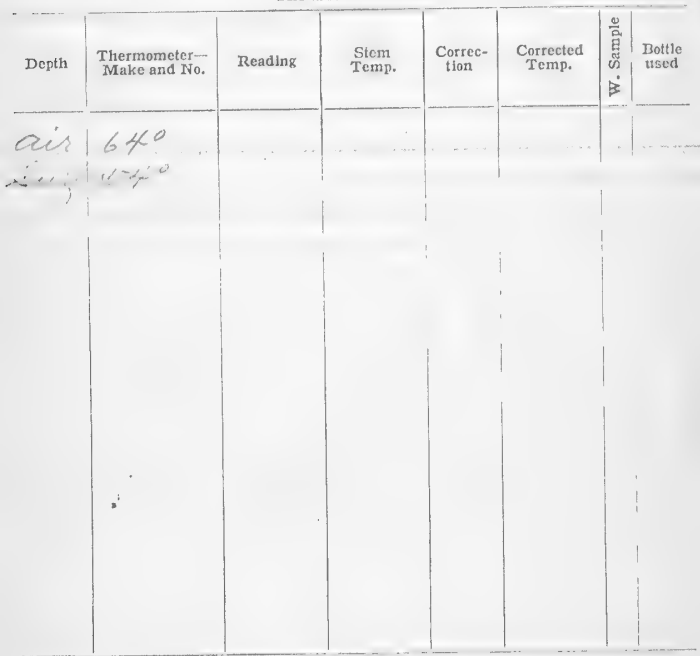

CURRENTS

\begin{tabular}{l|l|l|l|l|l|}
\hline Depth & Hour & Tide & Time dewn & Revolutions & Speed \\
\hline & & & & \\
& & & & \\
\end{tabular}

Color of water: 724

Transparency:

REMARKS:

$11-5314$ 
NETS, ETC , USED

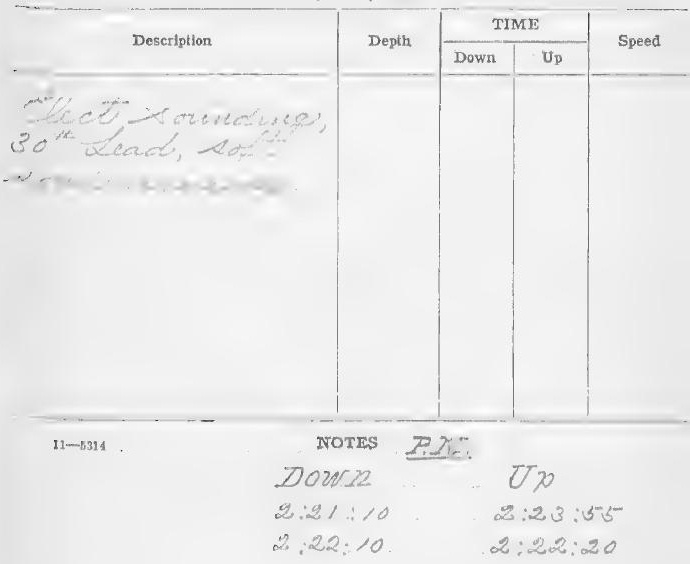

Porition: Tellaneo ot Head Laplet herso N590 E (o) (thue) risstrall 30.5 mine, 
Station No.

Date:

Hour: $2 ; 38: 5=0.2 . M$ to $2 ; 41: 65$ P. M.

Position: Lat.

Long.

\section{Locality:}

Depth: $14=\ldots$,

Bottom:

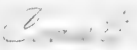

Wind: Direction, $\ldots, \sigma \ll ;:$ Force,

Sea: Cl. Sky: oreve.

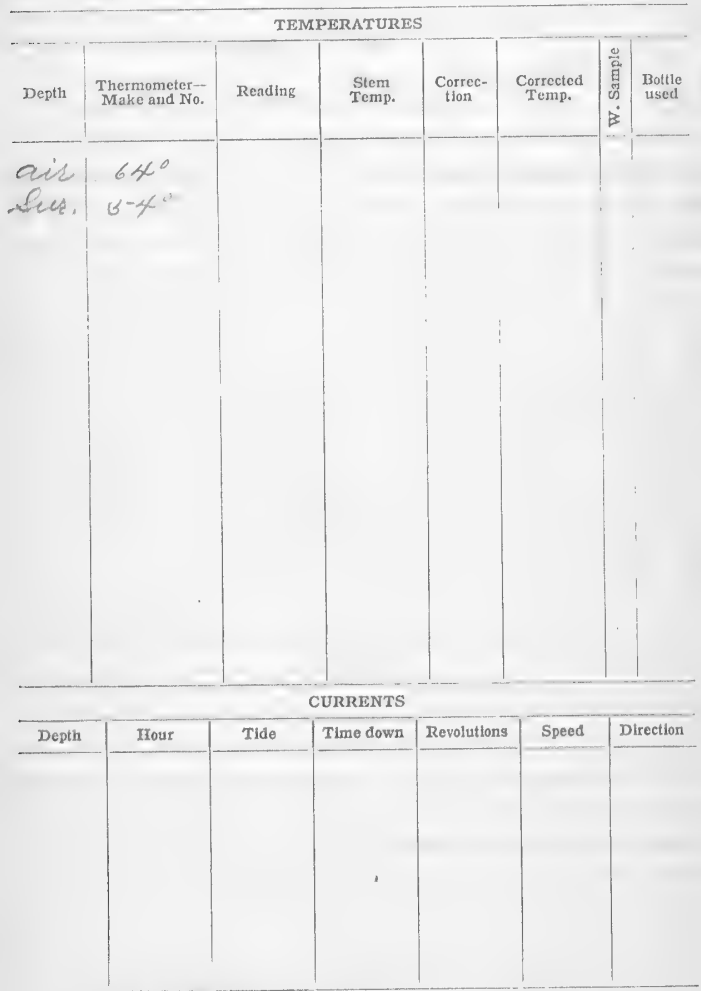

Color of water: in

Transparency:

REMARKS:

$11-\$ 314$

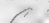

(P) 
Hour: $3: 34:$ to $P . M$. to $3: 3 \%, 50$ P.M.

Position: Lat.

Long.

\section{Locality:}

\section{Depth:} i. Bottom:

Sample:

Wind: Direction, Sowte Force, 3 .

\section{Sea:}

Siky: - feswu

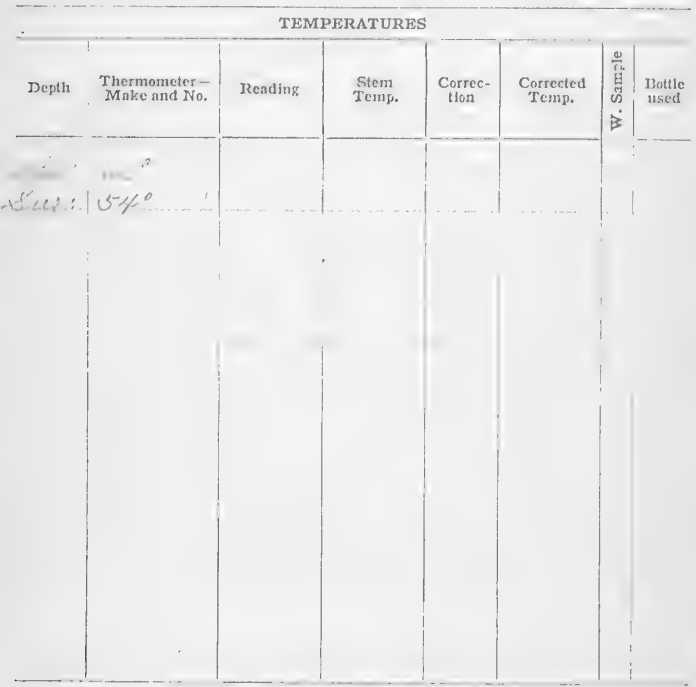

CURRENTS

\begin{tabular}{l|l|l|l|l|l|l|}
\hline Depth & Tide & Time down & Revolutions & Speed & Direction \\
\hline & & & & & \\
& & & & & \\
& & & & & \\
\hline Color of water: que & & & & \\
\hline
\end{tabular}

REMARKS:

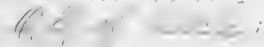


NITS, ETC., USIED

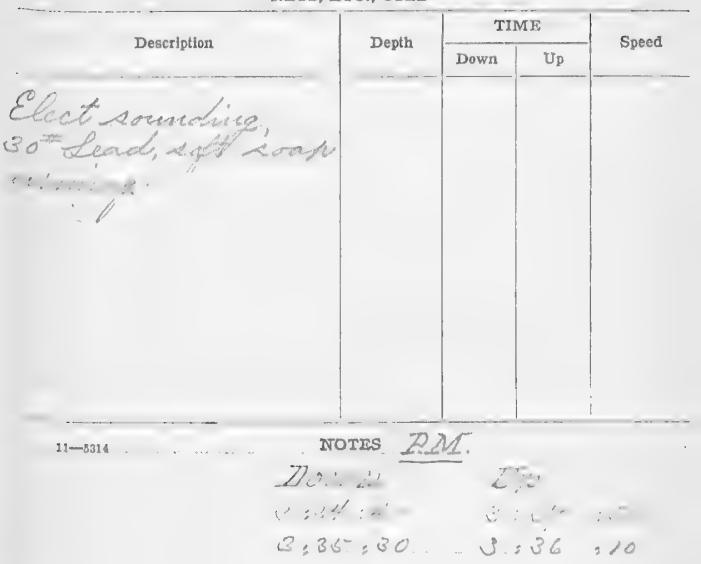

Position: Capendeares sifut heress $570^{\circ} \mathrm{E}$ (thus) Qhast 6100 
Hotr: $\angle)_{1} 3_{0}$ P.M. to $\angle, 1 L 20 \quad P . M$.

Position: Lat.

Long.

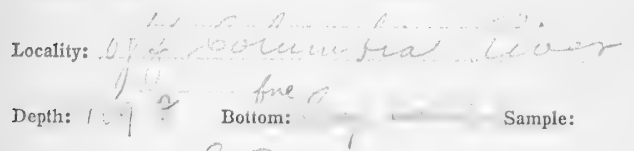

Wind: Direction,

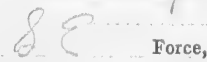

Sea:

Sky: $\quad$ -

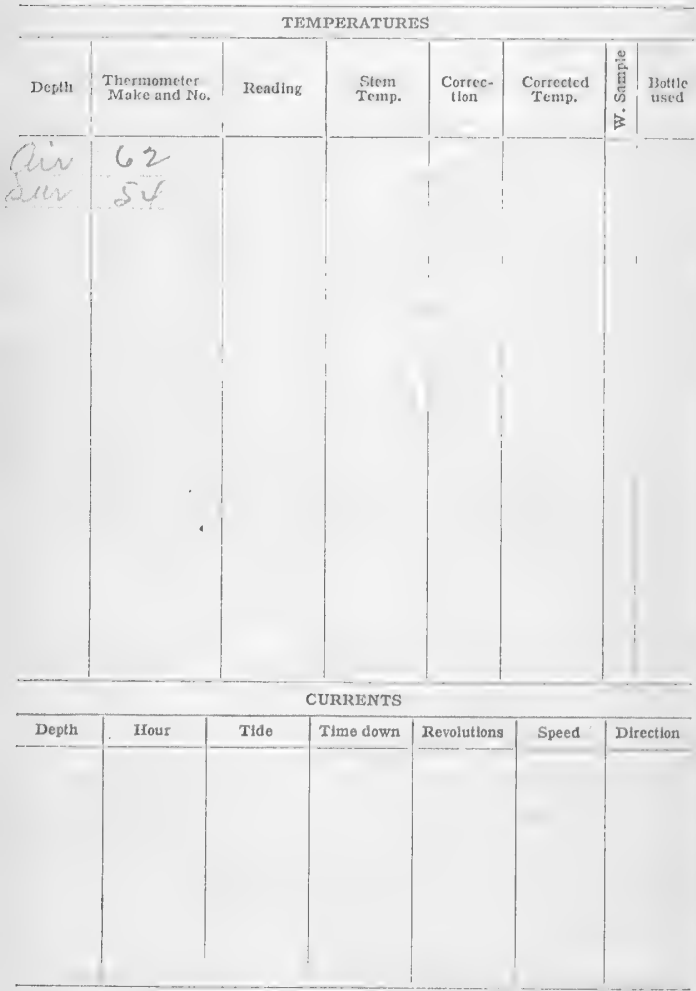

Transparency: 
NETS, ETC., USED

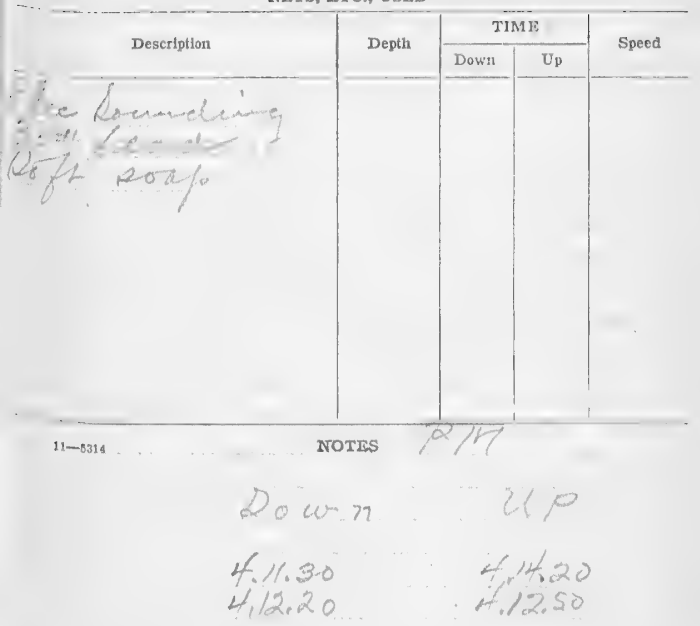

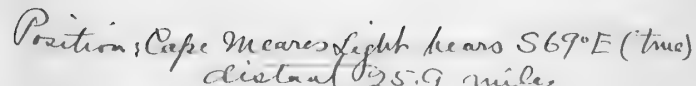
ohert b100 
Station No. $\%$ Date: $1901 \%$ Hour: $\mu_{\text {M. to }}$, i.

Position: Lat. Long.

\section{Locality:}

Depth: 104,2 is Bottom:,$\ldots \ldots$ Sample:

Wind: Direction, Force, . . F

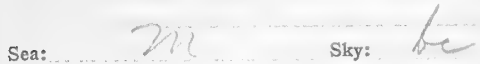

TEMPERATURES

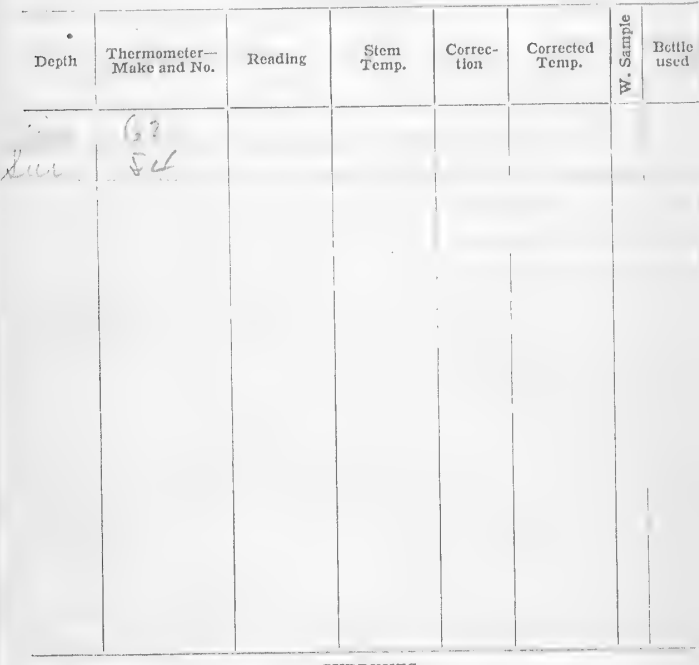

CURRENTS

\begin{tabular}{|c|c|c|c|c|c|c|}
\hline Depth & IIout & Tide & Time down & Revolutions & Speed & Direction \\
\hline & & & & & & \\
\hline & & & & & & \\
\hline & & & & & & \\
\hline & & & & & & \\
\hline & & & & & & \\
\hline
\end{tabular}


NETS, ETC., USED

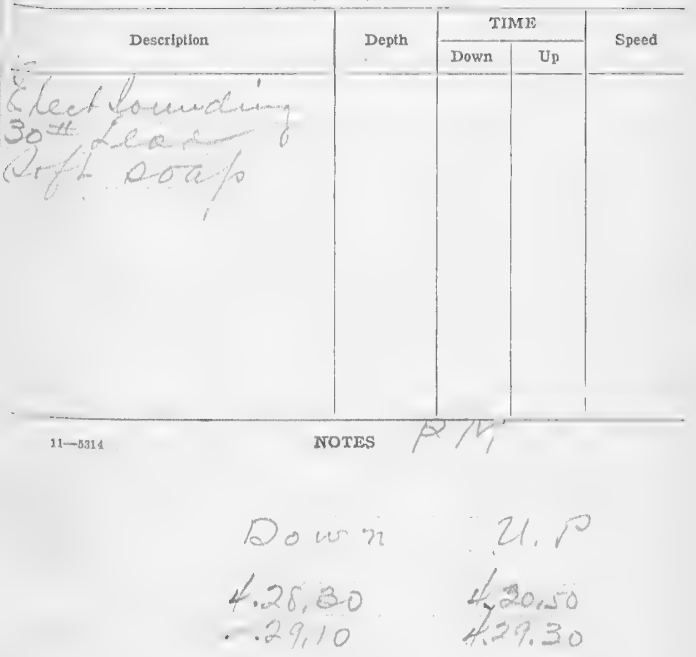

Pooctions Cape meares fight he ars S680 E (heme) chant 6100 diatant 24.5 mile. 
Hour:,$\ldots \ldots+$.

Position: Lat.

Long.

Locality:

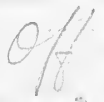

Depth: / . * , Bottom: Sample:

Wind: Direction, $\alpha \div$ fire gy $\leq$ Force,
Sea:
$2-22$
Sky:
$6 c$

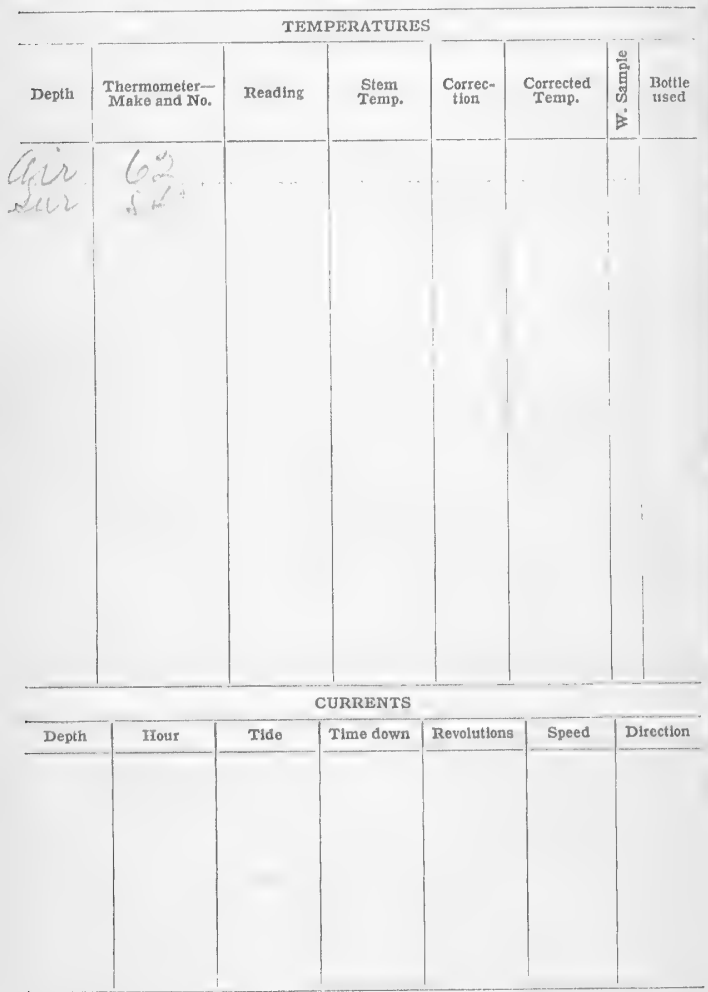


NETS, ETC., USED

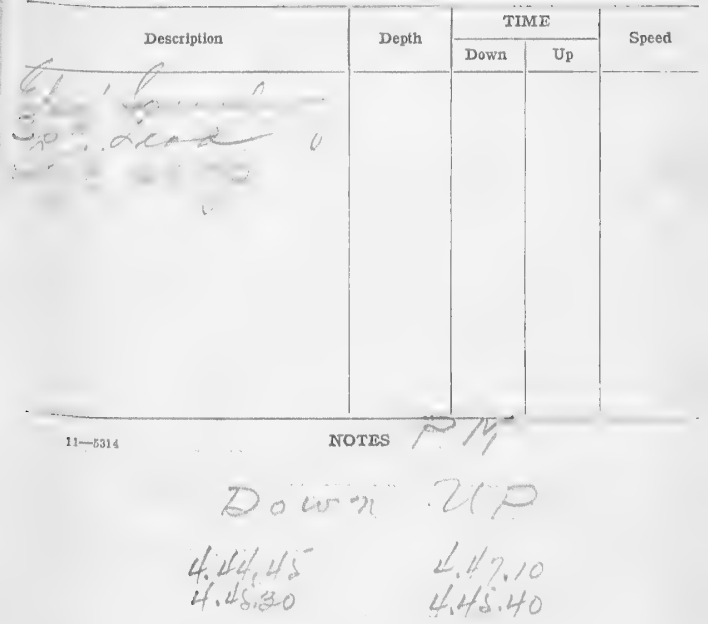

Position: Cape Inceares dight he ars $S 67 \% 2^{\circ}$ E (thers) chast 6100 diatred 23.2 m......, 
NETS, ETC., USED

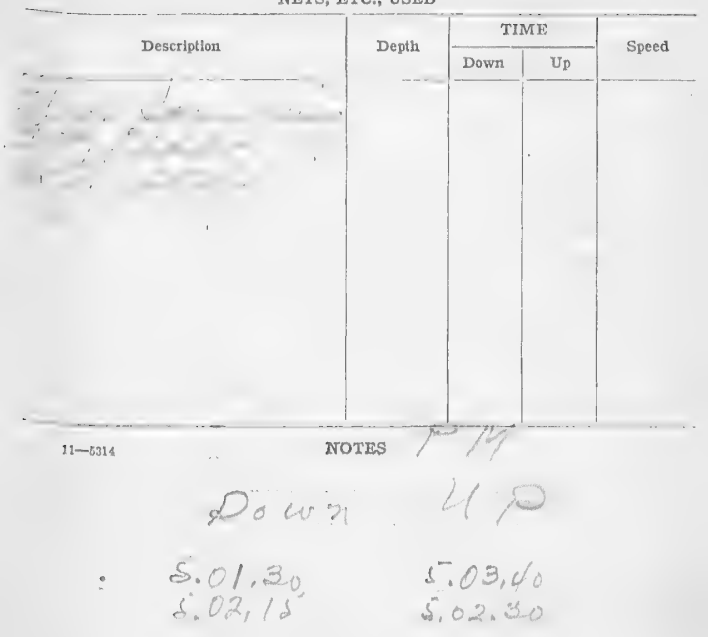

Porition; Cape mearas Light he aro $S\left(66^{\prime} / 20\right.$ E(tive) chert b/o0 


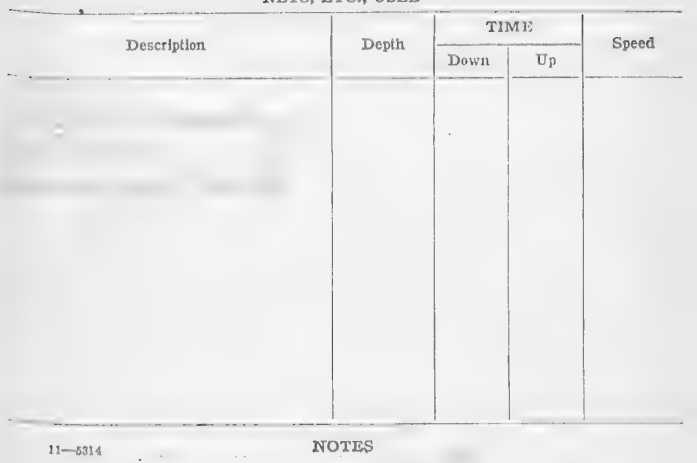

Poition: Cape enceares 2 githt bears $S 690$ E(then) Chenat 6/00 
Station No. Hour: $4, \ldots$, to $\ldots . . .$.

Position: Lat.

Long.

Locality:

Depth: Bottom: $\quad$ Bample:

Wind: Direction, $\ldots \ldots$, Force,

Sea:

Sky:

TEMPERATURES

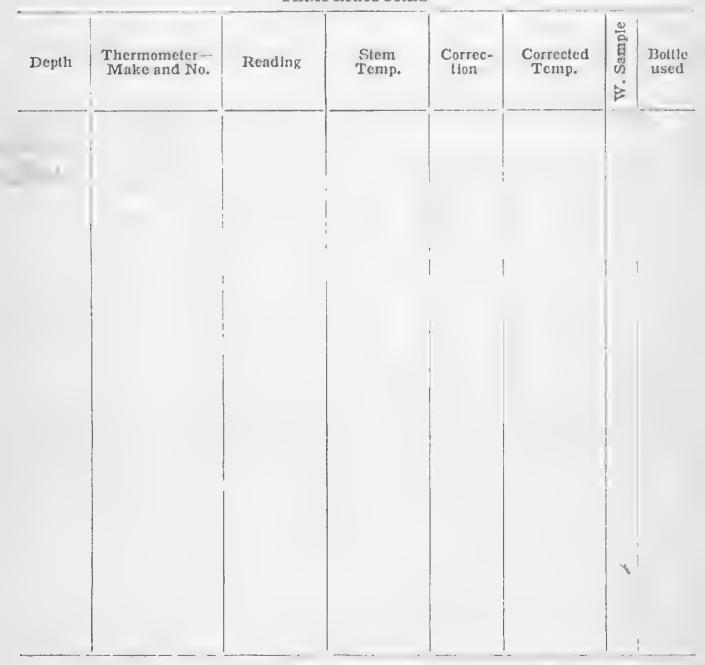

CURRENTS

\begin{tabular}{l|l|l|l|l|l|l}
\hline Depth & Tide & Time down & Revolutions & Speed & Direction \\
\hline & & & & \\
& & & & \\
& & & & \\
\end{tabular}




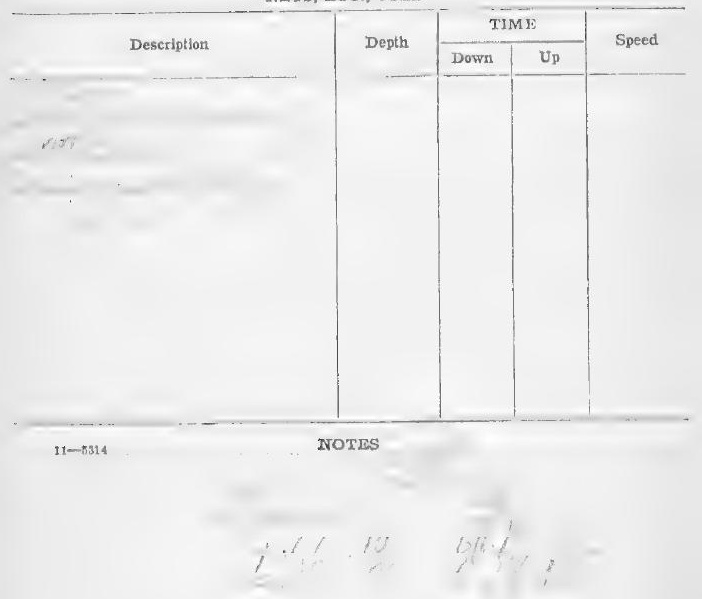

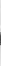
Chast 6100 distant 22.8 mies 


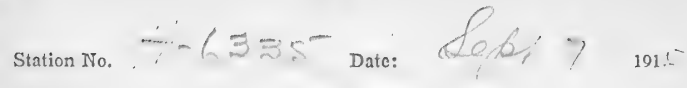
Hour: $2: 4$. M. to $36 \circ,: \mathbf{M}$.

Position: Lat.

Long.

Locality:

Depth: ... $\quad$ Bottom: fre gy
Wind: Direction, $\quad$ Force,

Sea:

Sky:

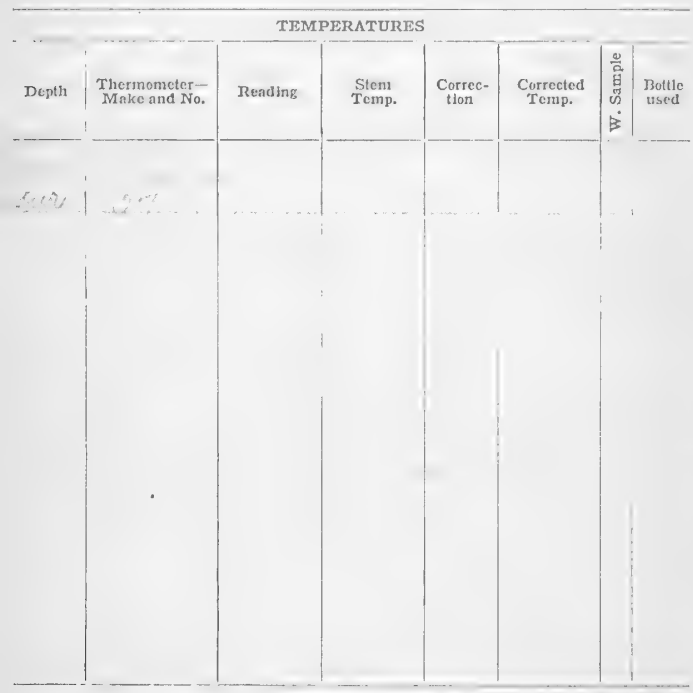

CURRENTS

\begin{tabular}{c|c|c|c|c|c|c|}
\hline Depth & Hour & Tide & Time down & Revolutions & Speed & Direction \\
\hline & & & & & \\
& & & & & \\
\end{tabular}


Station No. $t 6323$ Date: $206<>1914$

Hour: $2+30 \quad$, M. to

Position: Lat.

Long.

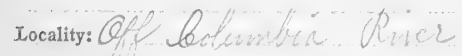

Depth: $f>$ Bottom: $\quad$ Sample:

Wind: Direction, Force,

Sea: _. Sky: $\quad \ldots \ldots \ldots$

TEMPERATURES

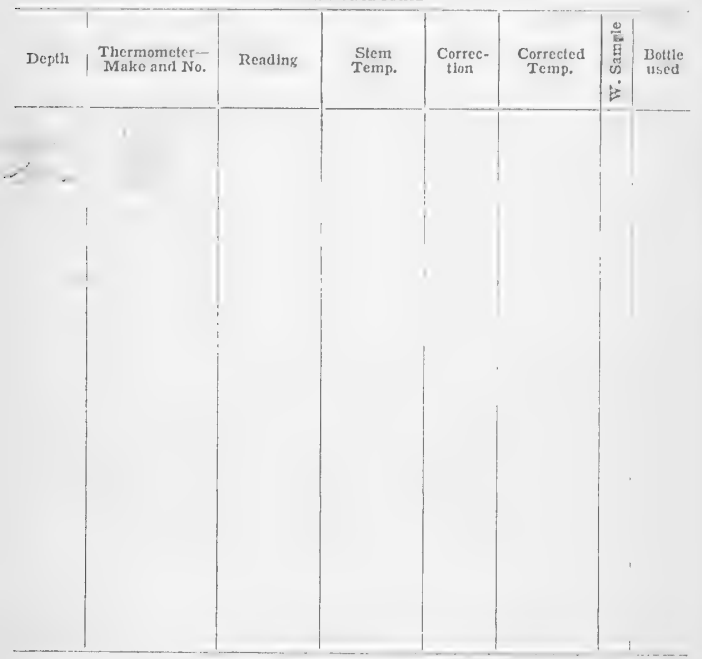

CURRENTS

\begin{tabular}{l|l|l|l|l|l|l}
\hline Depth & Hour & Tide & Time down & Revolutions & Speed & Direction \\
\hline & & & & & \\
& & & & & \\
\hline Color of water: & & & & \\
\hline REMARKS: & Transparency: & \\
\hline
\end{tabular}


NETS, ITC., USED

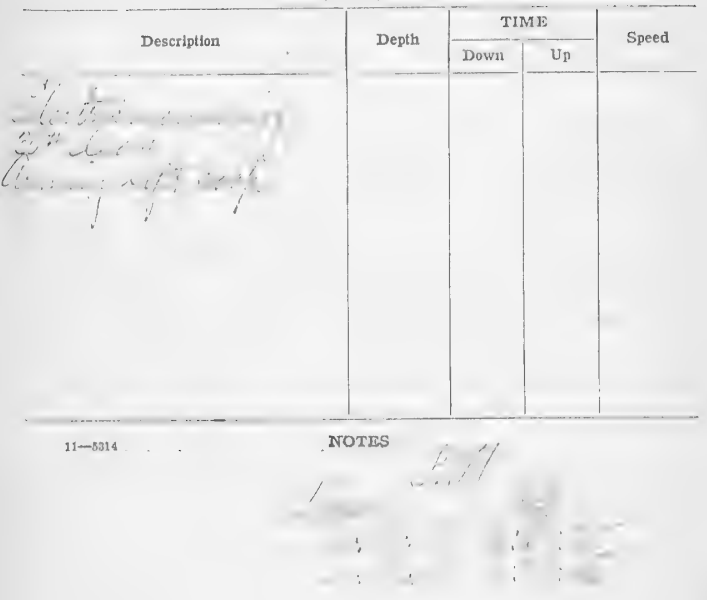

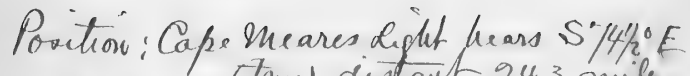
Dhast (thes) distant 24,3 mile 


\section{Station No. 46837 Date: $6015 \%$
M. to \\ Houx: \\ . M.}

Position: Lat.

Long.

\section{Locality:}

Depth: _... Bottom: Sample:

Wind: Direction,

Sea:

Sky: _fic

TEMPERATURES

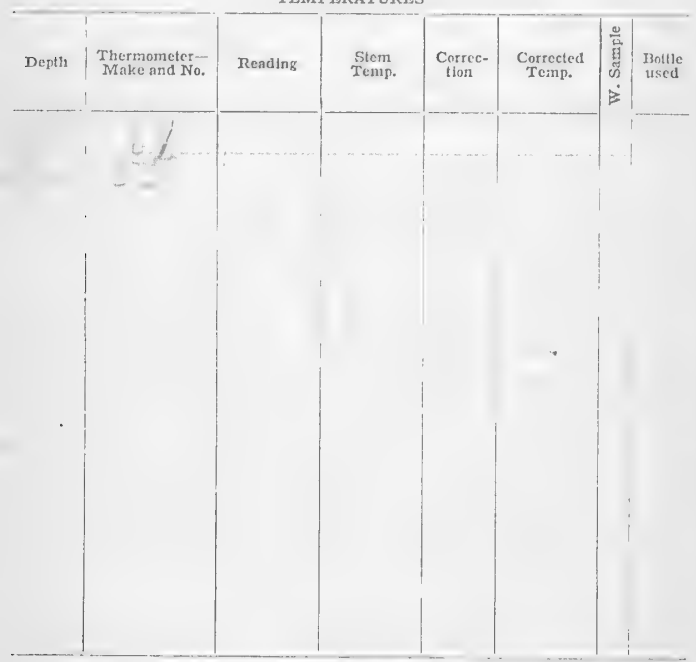

CURRENTS

\begin{tabular}{|c|c|c|c|c|c|c|}
\hline Depth & Hour & Tide & Time down & Revolutions & Speed & Direction \\
\hline & & & & & & \\
\hline & & & & & & \\
\hline & & & & & & \\
\hline & & & & & & \\
\hline & & & & & & \\
\hline
\end{tabular}

Color of water:

2. Transparency:

REMARKS:

$x+16$

$21-3314$ 
NRTS, ETC., USED

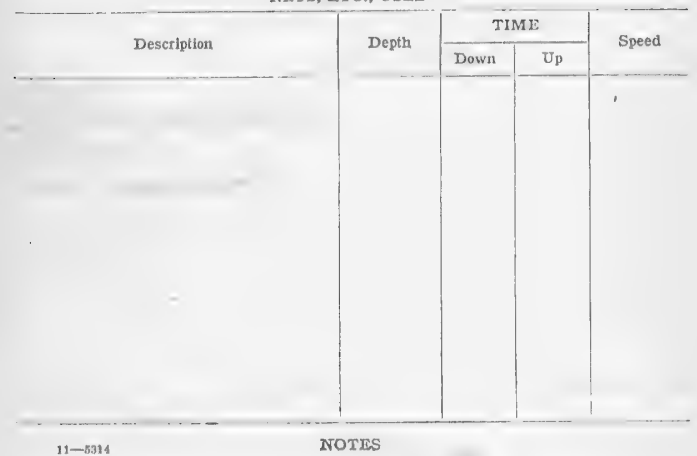

Poaction: Pepe Pneare. Lejut bears S\% $60 \mathrm{E}$ chart bioo (thue) distrat 25.0 ring... 
Station No.

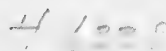

Date: ,

$191:$

Hour: $f, \ldots, \therefore$. to $f, j, \ldots$.

Position: Lat.

Long.

\section{Locality:}

Depth:

Bottom:

frie gy 3

Sample:

Wind: Direction,

Force,

Sea:

Sky:

TEMPERATURES

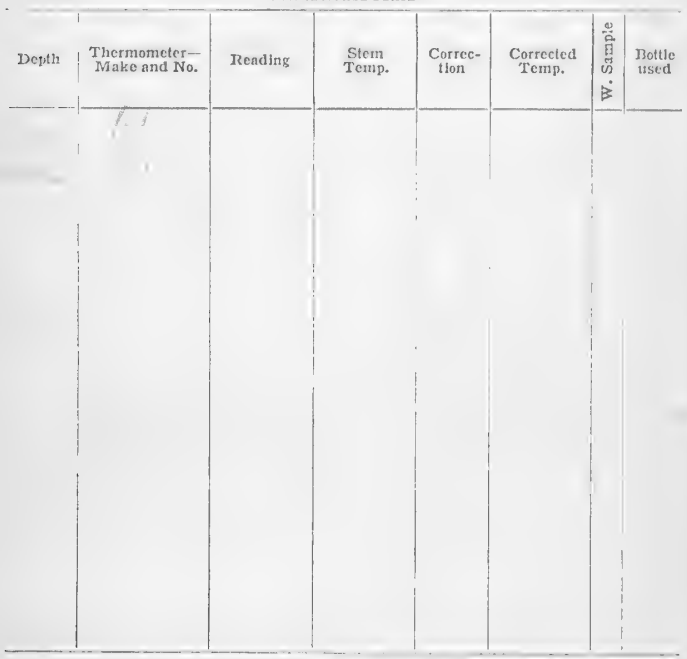

CURRIENTS

\begin{tabular}{c|c|c|c|c|c|c|}
\hline Depth & Hour & Tide & Time down & Rovolutions & Speed & Direction \\
\hline & & & & & \\
& & & & & \\
& & & & & & \\
\end{tabular}

Color of water:

Transparency:

REMARTS:

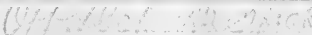

$11-\$ 314$ 


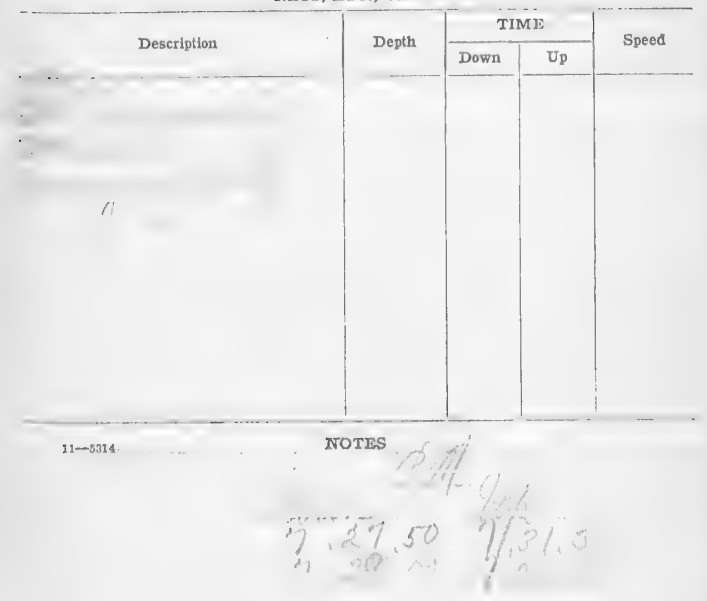

Position: Cope ineares dight hears $S 77^{\circ} \mathrm{E}$ chartbins 
Station No. $635 \%$ Date: $191=$

Hour: f:03iso .M. to fiog:to (.M.

Position: Lat.

Long.

Locality:

Depth:, Bottom:

Sample:

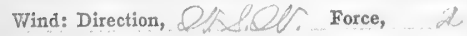

\section{Sea:}

Sky:

TEMPERATURES

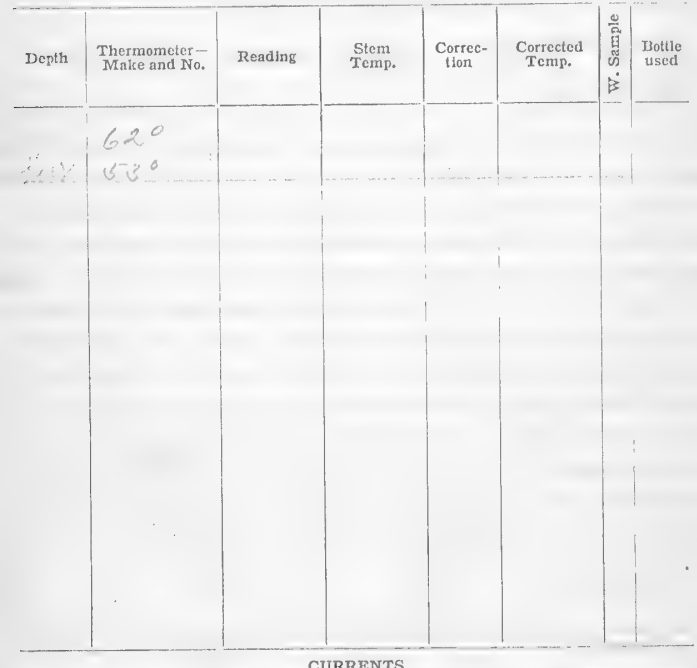

CURRENTS

\begin{tabular}{l|l|l|l|l|l|l|}
\hline Depth & Hour & Tide & Time down & Revolutions & Speed & Direction \\
\hline & & & & \\
\hline & & & & \\
& & & & \\
& & & & & \\
\end{tabular}

Transparency: 
NETS, ETC., USED

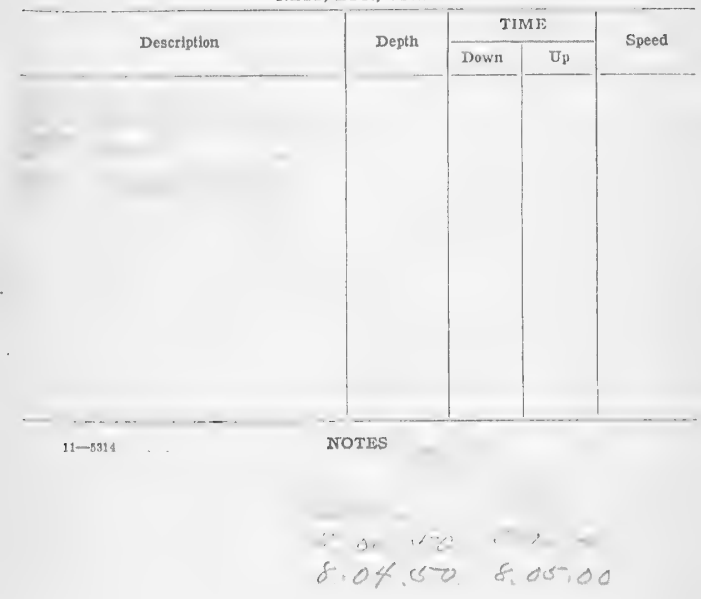

Porition: Cape encares fight he aro $577^{\circ} \mathrm{E}$ Qh (ture) distant 23.9 minle 
Station No. 1 ( 3 if Date: . . D

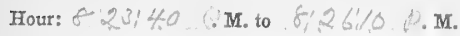

Position: Lat.

Long.

Locality:

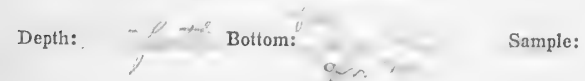

Wind: Direction, 2 fores 2
Sea:
Sky: -fe

TEMPERATURES

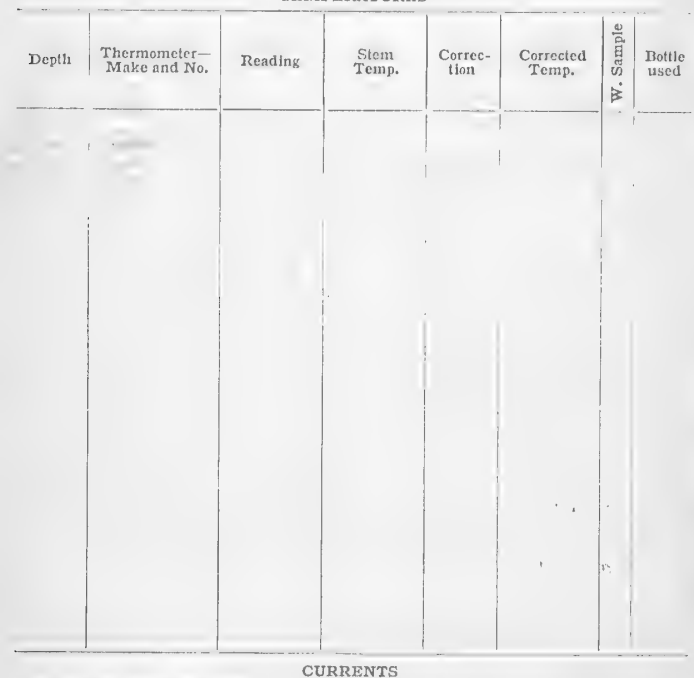

\begin{tabular}{l|l|l|l|l|l|l|}
\hline Depth & Tour & Tide & Time down & Revolutions & Speed & Direction \\
\hline & & & & & \\
& & & & & \\
& & & & & & \\
& & & & & \\
\end{tabular}

Transparency: 
NETS, ETC., USED

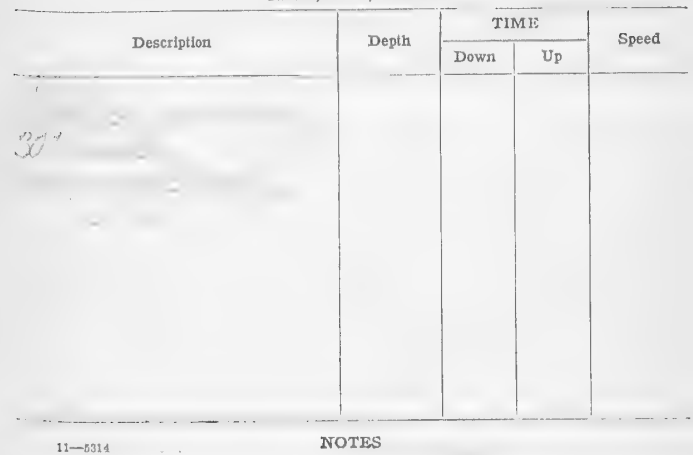

$$
\begin{aligned}
& 8: 23: 40 \text { 8:216:10 } \\
& 8: 24: 30 \text { 8:24:40 }
\end{aligned}
$$

Position: Dape meares dight hears $577^{\circ} \mathrm{E}$ (thus) distant 212.9 viciles chart 6100 


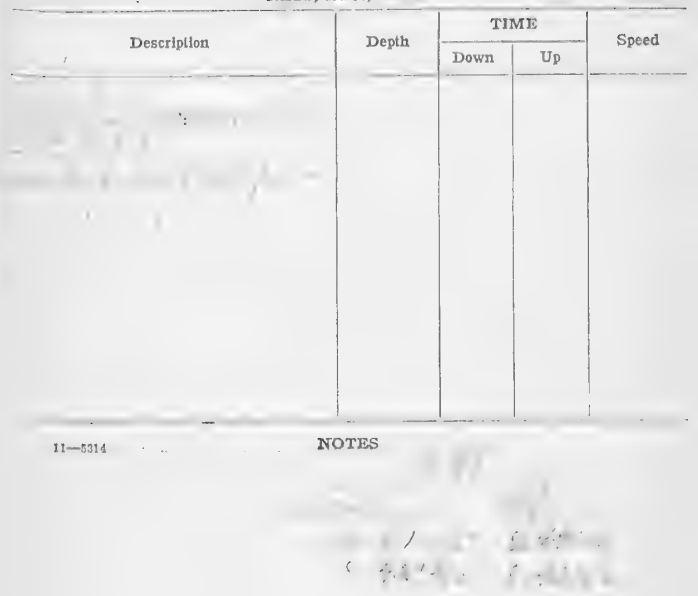

Position: Cape enceares ofyith hears $577^{\circ} \mathrm{E}$. chants 6/00 


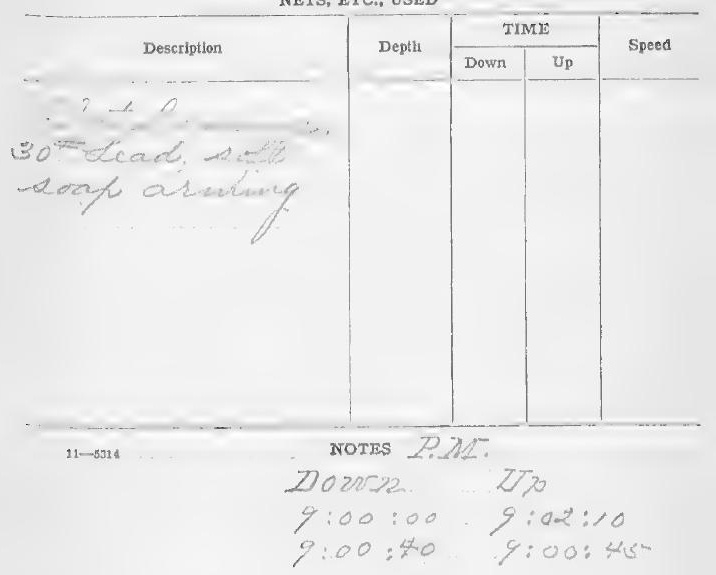

\footnotetext{
Poition: Papie sneares dight he ars $\$ 77^{\circ} \mathrm{E}$ chart the distonil 20.9 miks
} 


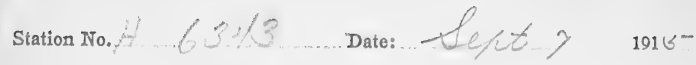

Hour: $q: 1, f: 5 \geqslant 0$, M. to $9: 20: 250 . M$.

Position: Lat. _................. Long.

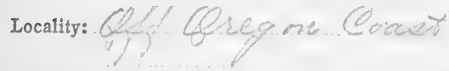

Depth: Thetom: fregres

Wind: Direction, PIA Pf, Force, $2 \ldots$

Sea:

Sky: Le

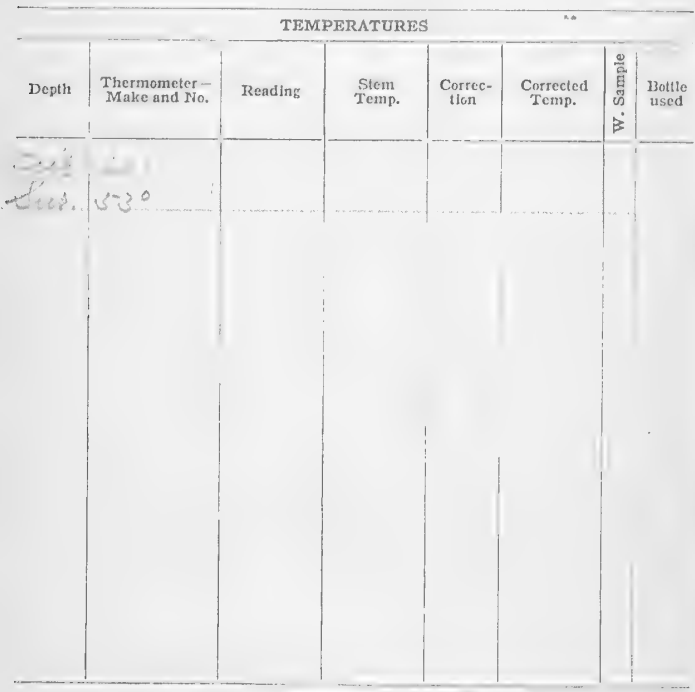

CURRENTS

\begin{tabular}{l|l|l|l|l|l}
\hline Depth & Tlour & Time down & Revolutions & Speed & Direction \\
\hline & & & & & \\
\hline & & & & & \\
& & & & &
\end{tabular}

Transparency: 
Station No.

Hour:

Position: Lat.

Long.

Locality:

Depth:

Bottom:

Sample:

Wind: Direction, $Y / . / Y, / / \ldots$ Force,

Sea: ... Sky:

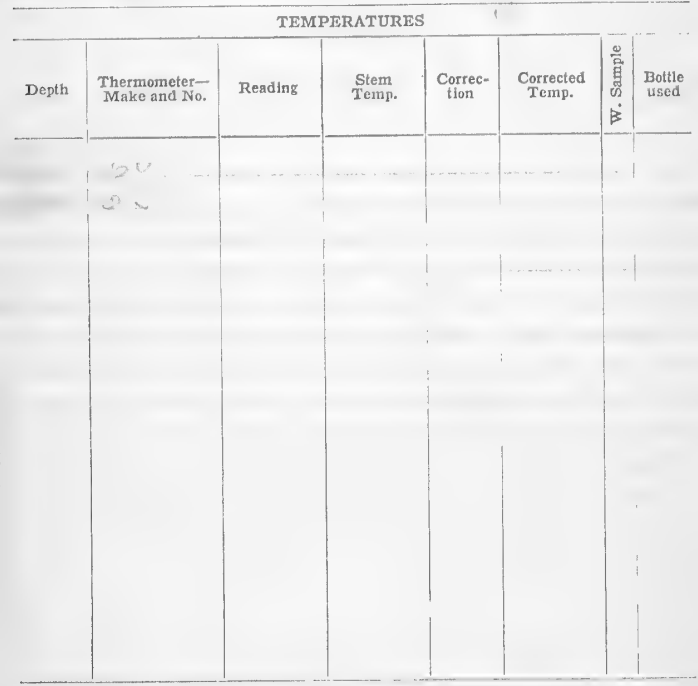

CURRENTS

\begin{tabular}{l|l|l|l|l|l|l}
\hline Depth & Thour & Tide & Time down & Revolutions & Speed & Direction \\
\hline & & & & & \\
\hline & & & & & \\
& & & & & & \\
& & & & & & \\
\end{tabular}


NETS, ETC., USED

$\left.\right|_{n=0} ^{5}$

Position: Cepe meares Light hears $584 \% 2^{\circ} \mathrm{E}$ (ture) distcht 17.4 necile, chant 6100 
NETS, ITC., USED

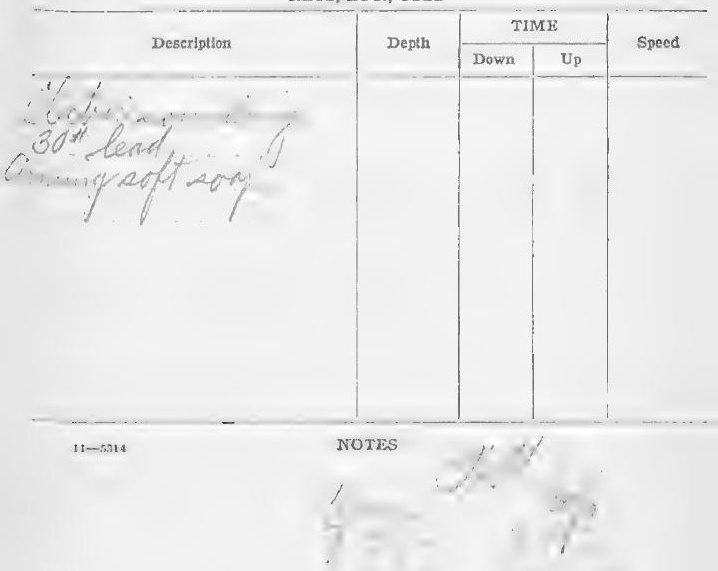

Position: Cope ancaresdight hears $585^{\circ} \mathrm{E}$ (twue) Chant diatand 18:4 mile. 


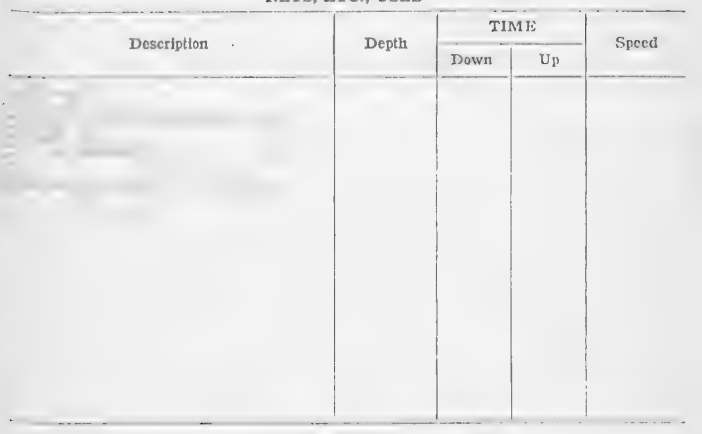

$$
\begin{array}{ll}
8,01,30 & 8.05,45 \\
8.02,10 & \cdots .2,15
\end{array}
$$

Position: Cope incares Leght heass $585^{\circ} \frac{1}{2} \mathrm{E}$ (hive) deot ant 19.4 suiles Qhaut Groo 


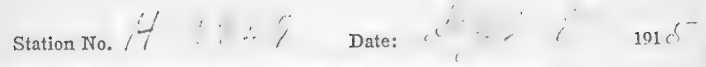
Hour: $\quad \bar{O} / . M$. to $(10$.

Position: Lat.

Long.

\section{Locality:}

$$
\text { C.t, }
$$

$$
\text { if }
$$

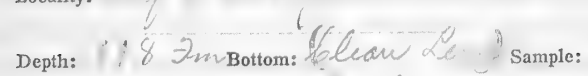

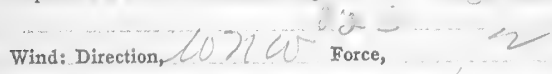

Sea:

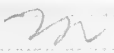

Sky:

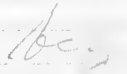

\section{TEMPERATURES}

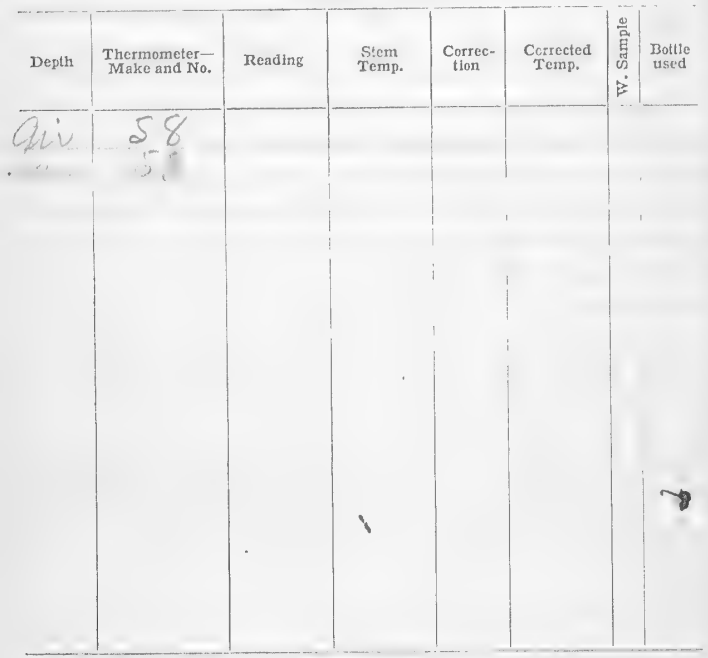

CURRENTS

\begin{tabular}{l|l|l|l|l|l}
\hline Depth & Tour & Time down & Revolutions & Speed & Directlon \\
\hline & & & & & \\
\hline & & & & &
\end{tabular}

Color of water:

Transparency:

REMARKS: 
Station INo.

Date:

1915

Hour: $\delta, 5,10 \quad \frac{A}{p} \mathbf{M}$. to $\delta, 5 j, \sigma 0,7 \cdot M$.

Position: Lat.

Long.

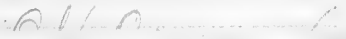

Locality:

Depth: $/ 1$, Bottom: Sample:

Wind: Direction, 107 Force,

Sea:

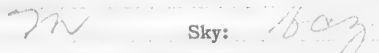

TEMPERATURES

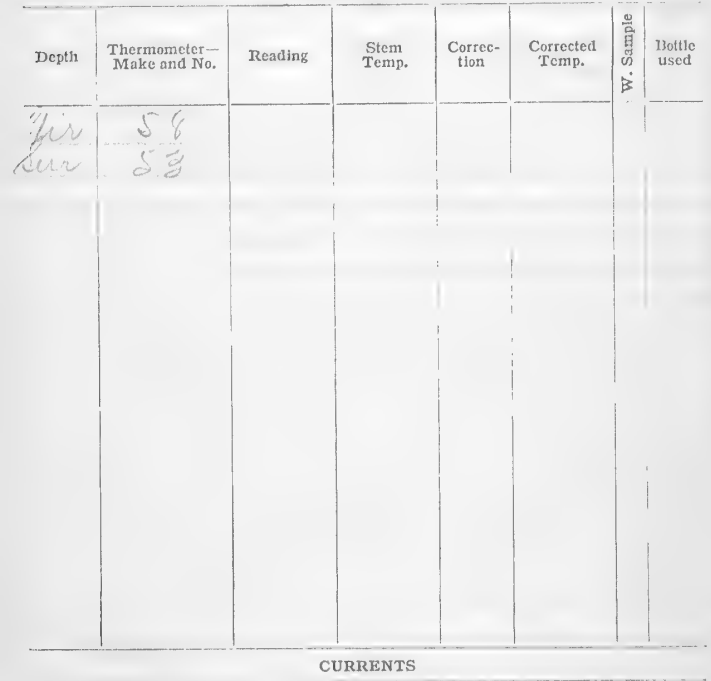

\begin{tabular}{l|l|l|l|l|l|l|}
\hline Depth & Thour & Tide & Time down & Revoiutions & Speed & Direction \\
\hline & & & & & \\
\hline & & & & & \\
& & & & & \\
\end{tabular}

Transparency:

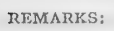


Station No. $:$, $/$ Date: $:=, \quad 1915$

Hour: COM. to

Position: Lat.

Long.

Locality: ( i celon cotot

Depth: 4 - 7 mBotom: 5 Sample:

Wind: Direction, (1) $\times$ h Force,

Sea:

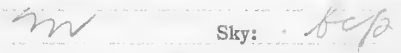

TEMPERATURES

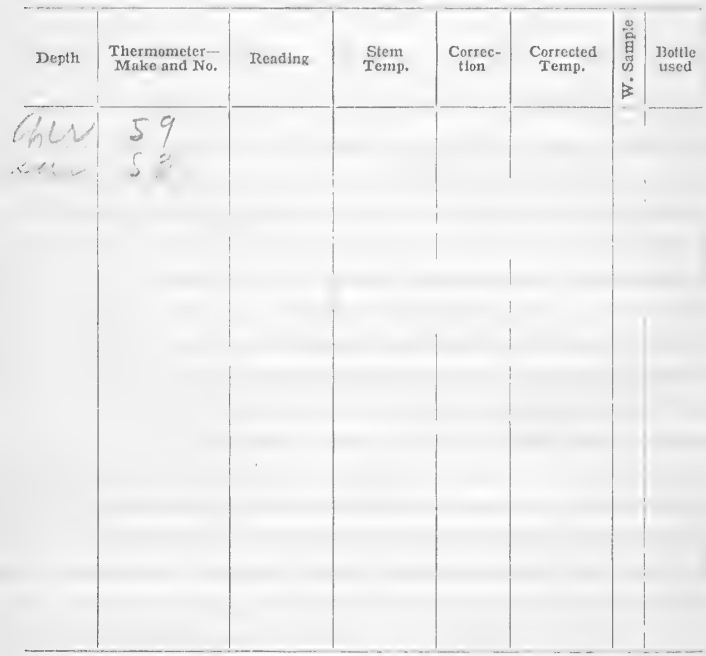

CURRENTS

\begin{tabular}{l|l|l|l|l|l|l|}
\hline Depth & Hour & Tide & Time dowr & Revolutions & Speed & Direction \\
\hline & & & & & \\
& & & & & \\
& & & & & & \\
\hline
\end{tabular}

Color of water:

Transparency: 
NETS, ITC., USED

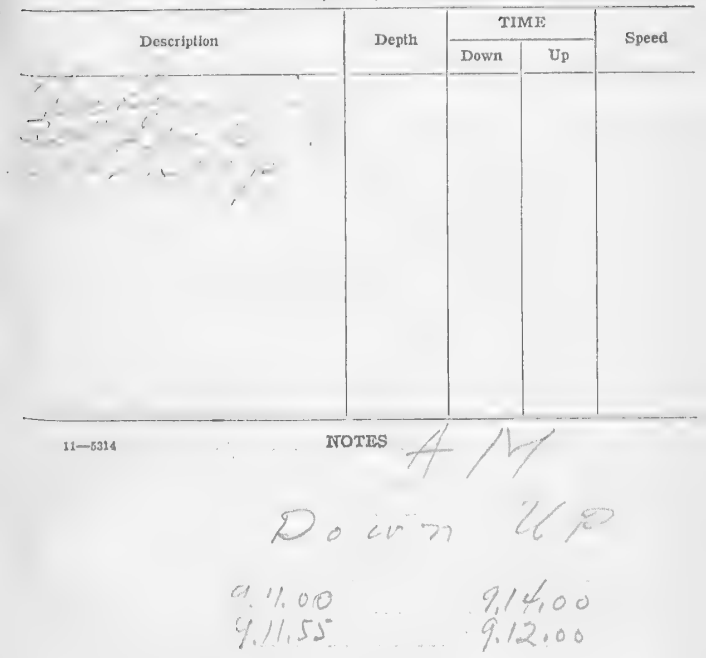

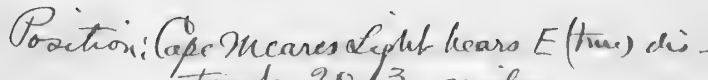
chat tant $20 / 3$ mit. chant 6100 


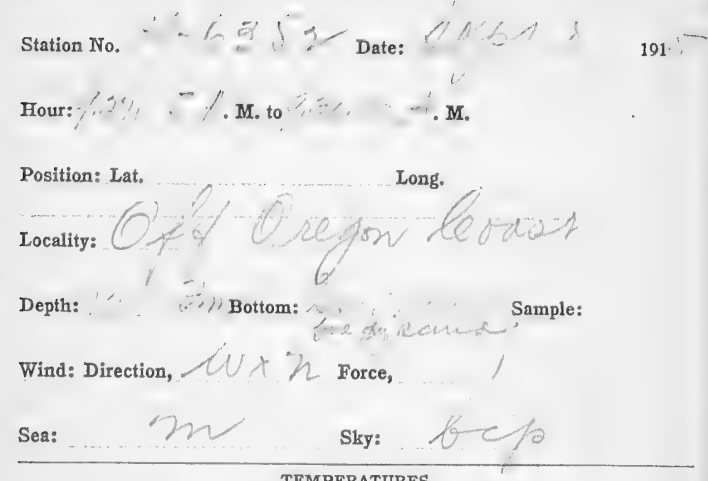

TEMPERATURES

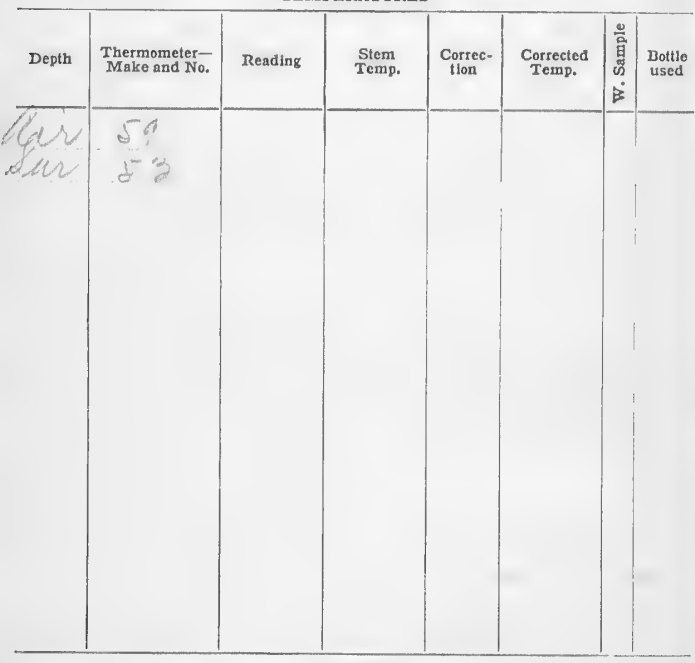

CURRENTS

\begin{tabular}{l|l|l|l|l|l|l|l}
\hline Depth & Tide & Time down & Revolutions & Speed & Direction \\
\hline & & & & & \\
\hline & & & & & \\
& & & & &
\end{tabular}

Transparency: 


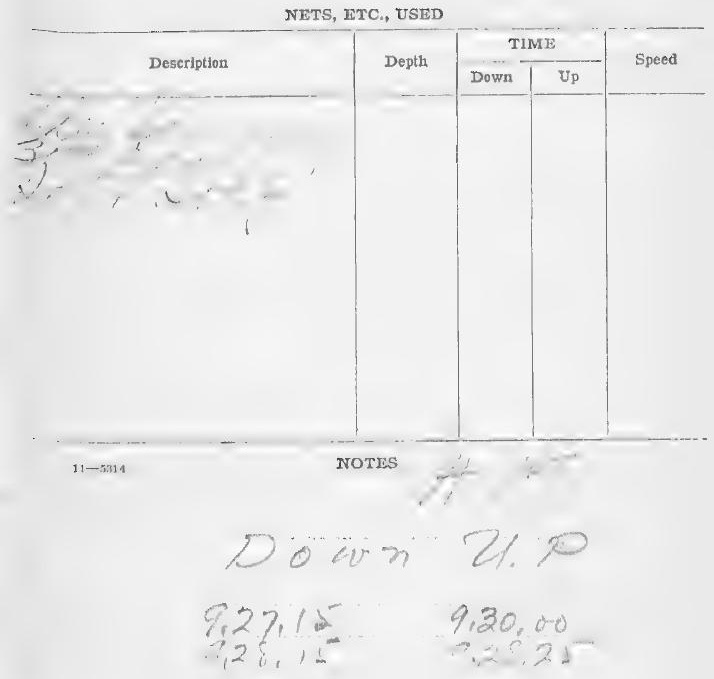

\footnotetext{
Positiou: Cape encares by ht hears $N 88^{\circ} \mathrm{E}$ (ture) disthent 19,2 mile Oaper corou
} 


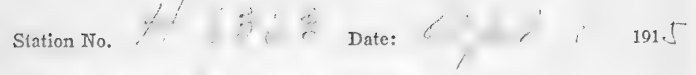
Hour: $i+2$ M. to $\therefore$ M.

Position: Lat.

Locality:

Depth: / / /

Wind: Direction, $2(1)+2$, Force,

Sea:

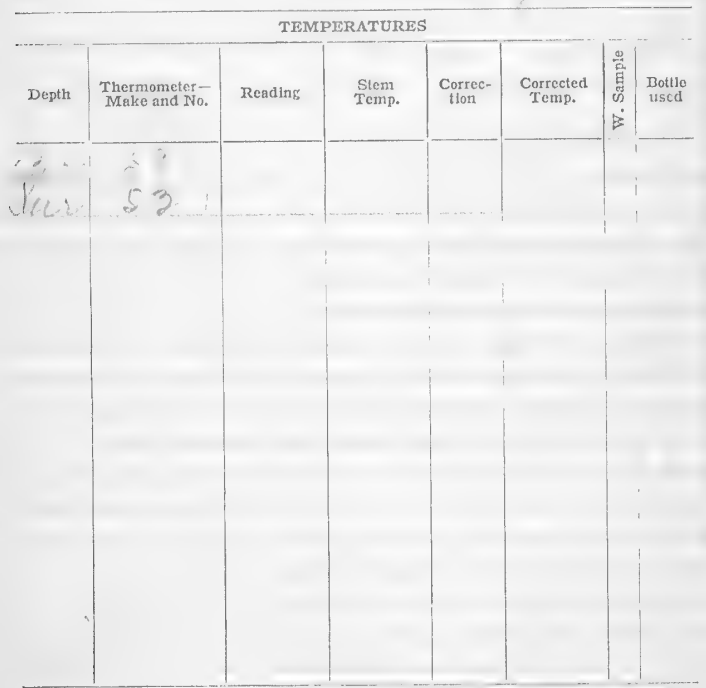

\section{CURRENTS}

\begin{tabular}{l|l|l|l|l|l|l}
\hline Depth & Tour & Tide & Time down & Revolutions & Speed & Direction \\
\hline & & & & & \\
& & & & & \\
& & & & & & \\
\end{tabular}

Color of water:

Transparency:

REMARKS:

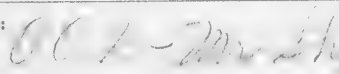

$11-5 n+14$
Sample:
Long. 


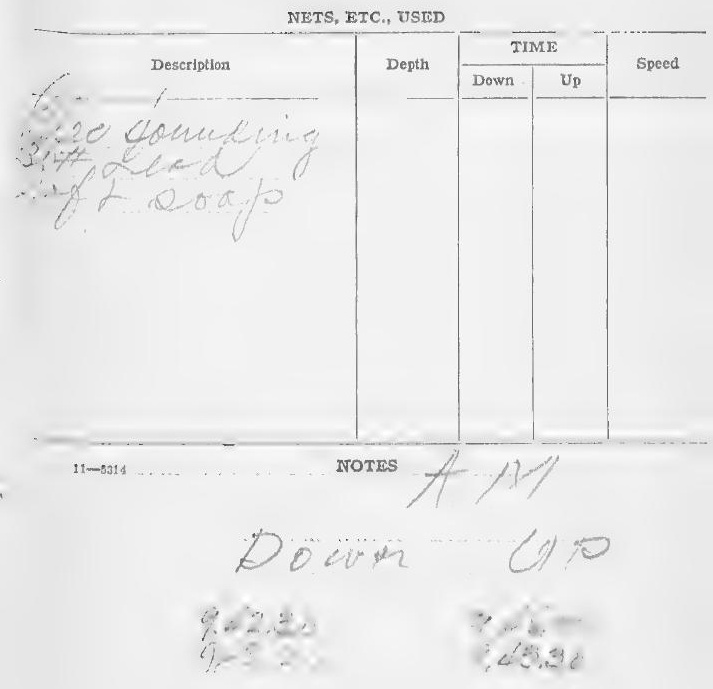

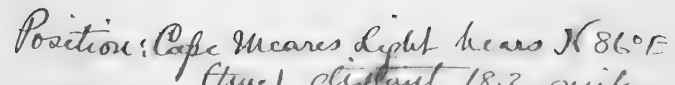

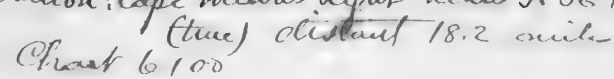


Station No. $\quad+1,+$ Date: 1915 Hour: $: \quad-$ M. to $\because 6: \div M$.

Position: Lat.

Long.

Locality:

Depth: $j$ Bottom: Sample:

Wind: Direction, $(1+)$ Force, ........

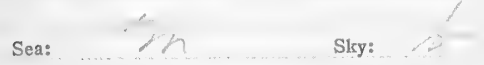

TEMPERATURES

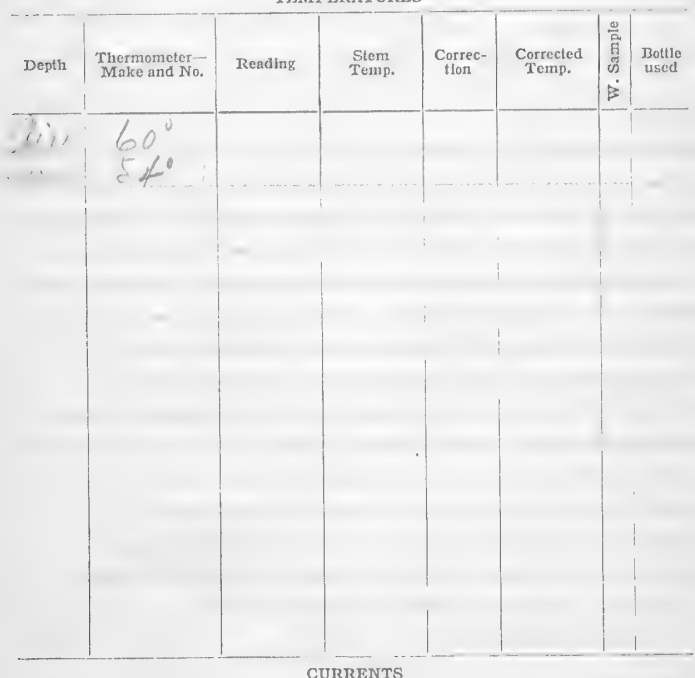

CURRFNTS

\begin{tabular}{l|l|l|l|l|l|l}
\hline Depth & Tour & Tide & Time down & Revolutions & Speed & Direction \\
\hline & & & & & \\
\hline & & & & & \\
& & & & & \\
\hline
\end{tabular}

Transparency:

REMARKS:

$11-5: 114$

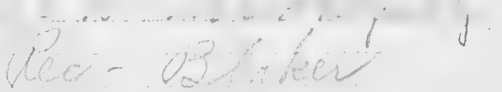


NETS, ITC., USED

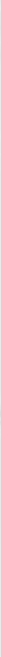

Position: Cope meares Light heurs N840 E

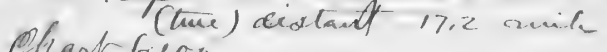
Chast le/ 100 
NETS, ETC., USED

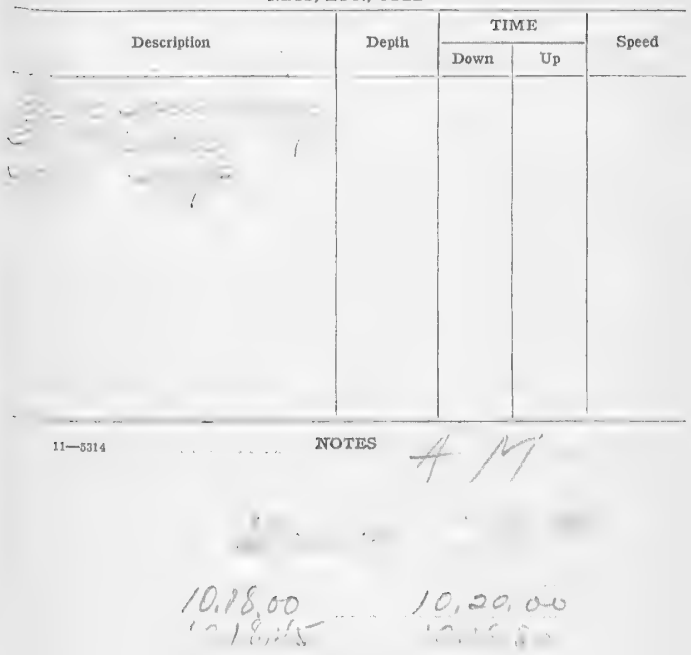

\footnotetext{
Porition: Bage nearus Lyut hears $N 82^{\circ}$ E (lin..) chart b100
} 
Station No.

Date:

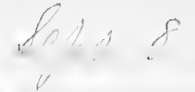

1915

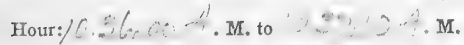

Position: Lat.

Locality:

Depth:
Wind: Direction, $/$ Sample:

Sea:

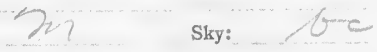

TEMPERATURES

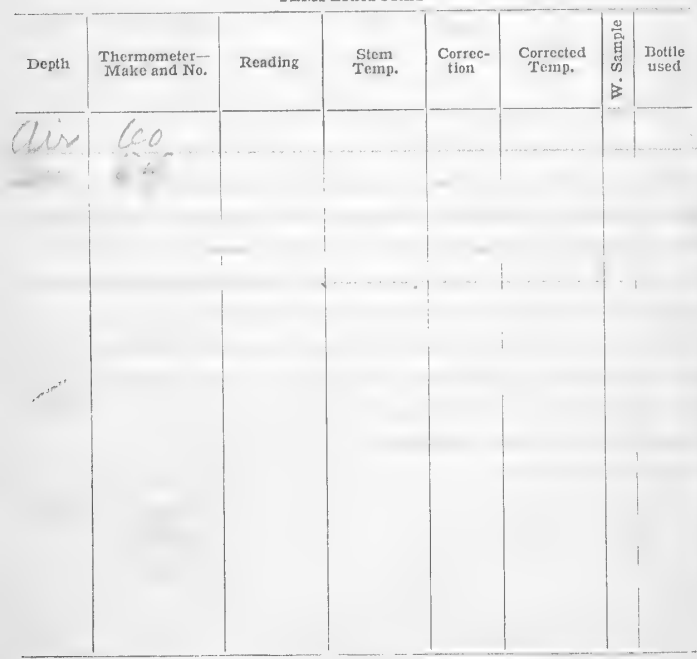

CURRENTS

\begin{tabular}{l|c|c|c|c|c|c}
\hline Depth & Hour & Tide & Time down & Revolutions & Speed & Direction \\
\hline & & & & & \\
& & & & & \\
& & & & & & \\
\end{tabular}

Color of water:

Transpaxency:

REMARKS:

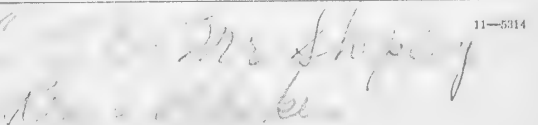




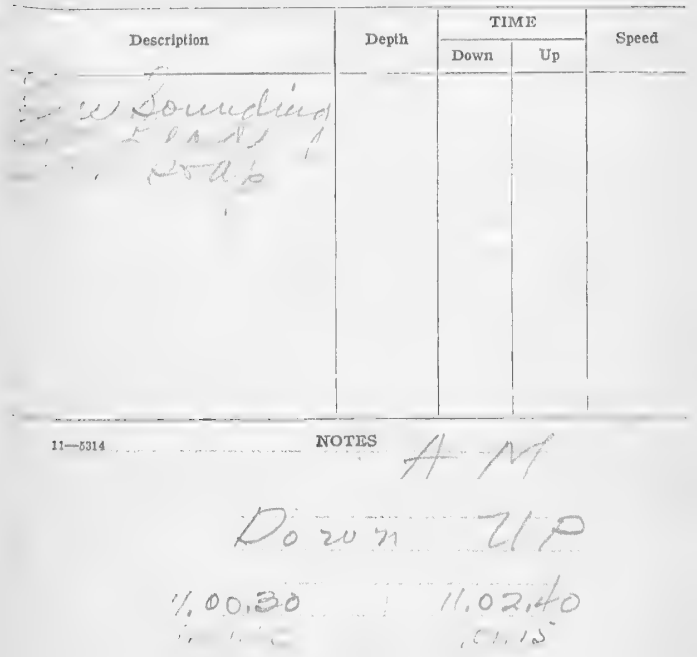

Position: Cape encares \& yith bears $N 77 \%$ (thec) distrubt 16 . smilo. chart 6100 
NETS, ITC, USED
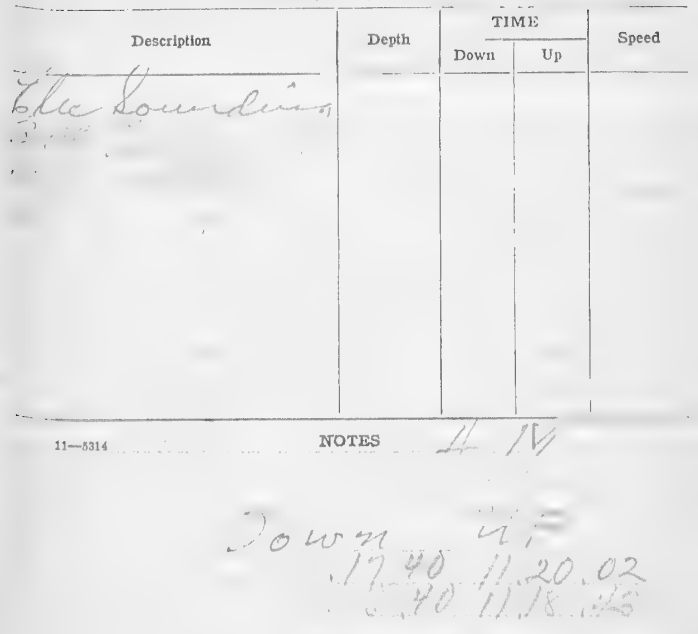

Poition: Cope queanes diaght hears N $75^{-3} \mathrm{k}^{6} \mathrm{E}$ chicest blove diotad 17,3 suik. 
Station No. 46359 Date: Sefa/ 8 191d Hour: $/ .35 .10$ A.M. to 1.37 .30 A. M.

Position: Lat. Long.

Locality: of Cugrow levart Depth: $117 \rightarrow m$ Bottom: Lelear le ad sample:

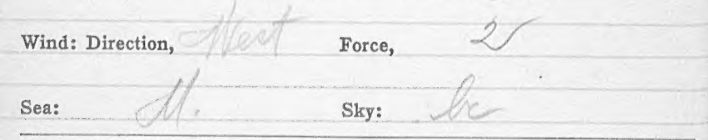

TEMPERATURES

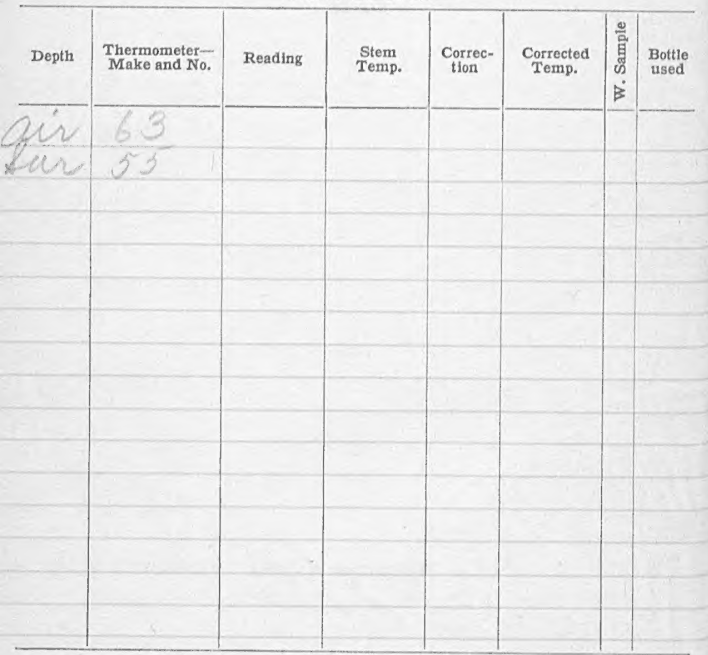

CURRENTS

\begin{tabular}{l|l|l|l|l|l|l}
\hline Depth & Hour & Tide & Time down & Revolutions & Speed & Direction \\
\hline & & & & & & \\
\hline & & & & & & \\
& & & & & & \\
& & & & & & \\
\hline
\end{tabular}

Color of water:

Transparency:

REMARKS:

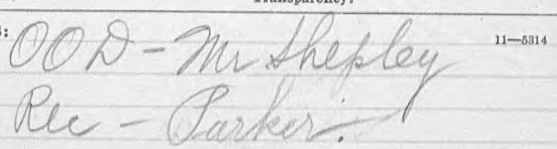


NETS, ETC., USED

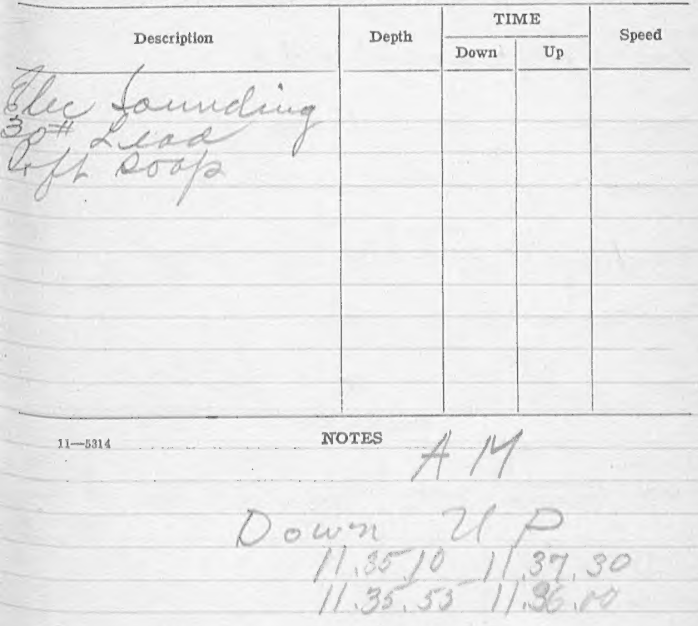

\section{Position: Cape meare dijut hears $\mathrm{N} 74^{\circ} \mathrm{E}$ (tsue) diatalut 18.3 suile chast b/ov}


16

station No. H 6360 Date: Rept 8

1915

Hour: $/ / 54,40$ A.м. to $/ / 56,50$ A.м.

Position: Lat.

Long.

Locality. If Oregon least

Depth: 148 2 $m$ in Bottom

Sample:

Wind: Direction, //en Force, 2

Sea:

Sky:

TEMPERATURES

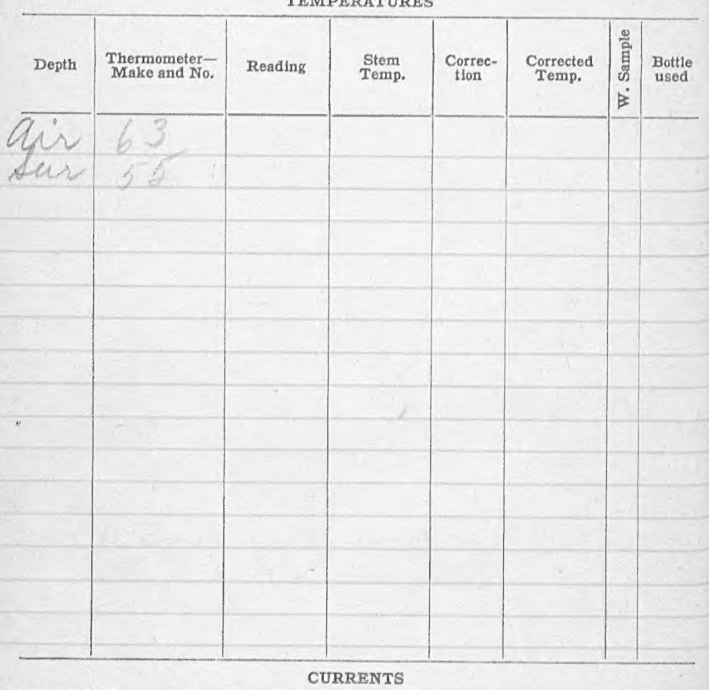

\begin{tabular}{l|l|l|l|l|l|l}
\hline Depth & Hour & Tide & Time down & Revolutions & Speed & Direction \\
\hline & & & & & & \\
\hline & & & & & \\
& & & & & & \\
\hline
\end{tabular}

Color of water:

Transparency:

REMARKS:

O ON- MW thepley "-m"

Re e-

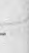


NETS, ETC., USED

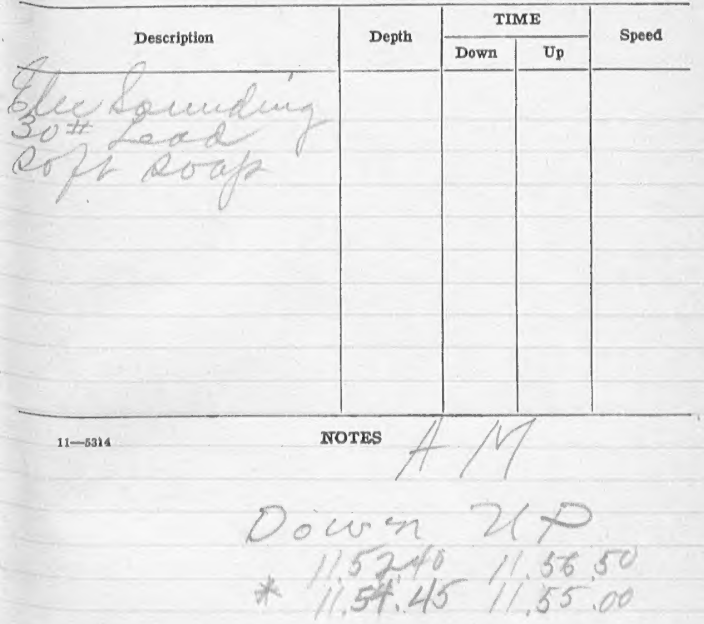

Position: Cape Mneares Light hearo $N 72 \%{ }^{\circ} \mathrm{E}$ (trox) diathent 19.4 miles chart 6100

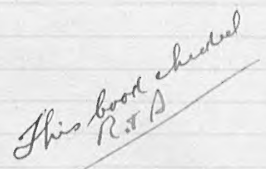

